\title{
The Hamiltonian Structure of Nonlinear Elasticity: The Material and Convective Representations of Solids, Rods, and Plates
}

\author{
Juan C. Simo \\ Division of Applied Mechanics \\ Stanford University \\ California
}

\author{
Jerrold E. Marsden \\ Control and Dynamical Systems \\ Caltech 104-44 \\ Pasadena, CA 91125 \\ marsden@cds.caltech.edu
}

\author{
P.S. Krishnaprasad \\ Department of Electrical Engineering \\ and Systems Research Center \\ University of Maryland \\ College Park, Maryland \\ version: July 19, 1995 \\ Communicated by S. Antman
}

\section{Contents}

1 Introduction 1

2 Covariant Three-Dimensional Elasticity 3

3 The Hamiltonian Structure of Three-Dimensional Elasticity in the Material and $\begin{array}{ll}\text { Convective Representations } & 15\end{array}$

\section{Introduction}

It is our belief that a thorough understanding of the mathematical underpinnings of elasticity is crucial to its analytical and numerical implementation. For example, in the analysis of rotating structures, the coupling of the equations for geometrically inexact models, obtained by linearization or other approximations, with those for rotating rigid bodies can easily lead to misleading artificial "softening" effects that can significantly alter numerical results; see Simo and VuQuoc [1986c] (especially equations (3) and (5)). In this paper, we consider fully nonlinear geometrically exact models for rods, plates (and shells) which take into account shear and torsion as well as the usual bending effects in traditional rod and plate models. These models can be obtained either from the three-dimensional theory by a systematic use of projection methods; see e.g., Antman [1972] and Naghdi [1972], or by a direct approach within the context of Cosserat continuum. Remarkably, the two approaches lead to essentially the same form of the governing field equations. In the present context, we have chosen as a model problem a particular rod model which may be regarded as an extension of the classical Kirchhoff-Love model (see Love [1944]) to include shear deformations, as in 
Reissner [1973, 1981], Antman [1974], Antman and Jordan [1975], and Simo [1985]. The counterpart of this model for plates is also considered. Our objective is a systematic development of the Hamiltonian structure underlying the dynamics of these geometrically exact models in the material and convective representations. The convective representation, which is often referred to as the body representation in the context of rigid-body mechanics, is a useful counterpart to the more familiar material and spatial representations. Understanding the relation between these alternative representations is useful for computational purposes and for coupling of these models to the dynamics of rigid body motion, as in Krishnaprasad and Marsden [1987]. We confine our attention to the material and convective representations only for simplicity; one can also treat the spatial representation and in fact we shall use the latter in a subsequent work on stability of rigid bodies with geometrically exact flexible attachments (see Krishnaprasad, Marsden, Posbergh, and Simo [1988]).

One of the topics that is of importance in the foundations of elasticity is a geometric formulation of the equations in Hamiltonian form. This form is useful in the dynamical analysis of systems; for example in the study of nonlinear stability (see Holm, Marsden, Ratiu, and Weinstein [1985], Krishnaprasad [1985], and Lewis, Marsden, and Ratiu [1986a]), of bifurcation theory (see Golubitsky and Stewart [1986] and Lewis, Marsden and Ratiu [1986b]) and of chaotic solutions (see Holmes and Marsden [1983] and Guckenheimer and Holmes [1983]). Our own motivation is to provide additional insight for work on rotating structures using geometrically exact models (see Krishnaprasad and Marsden [1987] and Krishnaprasad, Marsden, Posbergh and Simo [1988]). Of course, independent of this motivation is the fact that these Hamiltonian structures are of intrinsic interest for the mathematical foundations of elasticity theory.

The Hamiltonian structure for the material (or Lagrangian) representation of elasticity is given in terms of canonically conjugate variables - namely the placement field and its conjugate momentum, the momentum density. This standard result is well known and is indicated in, for example, Marsden and Hughes [1983, Chapter 5]. The relation between this and other structures in spatial and body representations is an important result that goes back to Arnold [1966] and was developed by Marsden and Weinstein [1974, 1982], and others. A noncanonical Hamiltonian structure for elasticity that is partially a spatial representation is given in Holm and Kuperschmit [1983], and a Hamiltonian structure for isotropic elasticity in spatial representation is given in Marsden, Ratiu, and Weinstein [1984a,b]. We deal with these as well as the convective representation and also develop a Hamiltonian formalism for rods and plates. A geometric setting that is useful for understanding the general relation between the material, inverse material, spatial, and convective representations, and relies partially on the present work is given in Holm, Marsden and Ratiu [1986].

The noncanonical brackets found in this paper are obtained by the general methods of reduction from the canonical structure in material representation, as in Arnold [1966] and Marsden and Weinstein [1982]. When these procedures are done for fixed boundary problems, one obtains Lie-Poisson brackets associated with the dual of a Lie algebra of a semi-direct product. (See Marsden, Weinstein, Ratiu, Schmit and Spencer [1983] for a general introduction to this geometric theory.) These sorts of brackets appear for example in the equations for compressible fluids and magnetohydrodynamics (see Marsden [1982], Holm and Kuperschmit [1983] and Marsden, Ratiu and Weinstein [1984a,b]). (The geometric reason for this appearance is that if a configuration space is a group $G$, then the reduction of the phase space $T^{*} G$ by the isotropy subgroup $G_{a}$ for a representation of $G$ on a vector space $\mathbf{V}$ is essentially the dual of the Lie algebra of the semi-direct product $G$ ? $\mathbf{V}$. This result, due to Ratiu, Guillemin and Sternberg, is proved in a sharpened version in Marsden, Ratiu and Weinstein [1984a] (to which we refer for the original references). When free boundaries are present, however, the brackets are only partially of the Lie-Poisson type. The geometric setting for these is the "gauged Lie-Poisson" context of Montgomery, Marsden and Ratiu [1984]. This was applied to free boundary fluid problems in Lewis, Marsden, Montgomery and Ratiu [1986]. In this paper, we shall not require the fairly sophisticated context of the gauged Lie-Poisson structures, but rather we shall obtain the results by a direct calculation. We do note, however, that when no boundaries are present, the Poisson brackets we get for three dimensional elasticity do reduce to Lie-Poisson 
brackets for a semidirect product. For rods and plates, the brackets also reduce to the Lie-Poisson type in the cases that the configuration space reduces to a group; for example, this happens for the torsional motion of a rod.

The geometric point of view adopted in this paper has proven particularly useful in the numerical solution of initial boundary value problems. For the geometrically exact rod model, for instance, exact update procedures for the configuration, stress resultants and stress couples can be developed by employing discrete algorithmic counterparts of the exponential map and parallel transport (see Simo and Vu-Quoc [1986a,b, 1987]). These ideas also play a central role in the numerical analysis of geometrically exact shell models, as in Simo and Fox [1988] and Simo, Fox and Ritai [1988a,b]. This methodology results in algorithms that exactly preserve the fundamental physical requirement of material frame-indifference. Similarly, for three-dimensional nonlinear viscoelastic solids, by exploiting the convective representation, one can develop unconditionally stable algorithms, accurate to second order, which exactly preserve covariance of the continuum formulation (see Simo [1987]). Thus, these algorithms go beyond the notion of incremental objectivity, as proposed by Hughes and Winget [1980]. Finally, we believe that the Hamiltonian structures developed in this paper will play a central role in the future development, design and stability analysis of time-stepping integration algorithms for nonlinear elastodynamics, which ensure not only conservation of energy (as in Chorin, Hughes, McCracken and Marsden [1978] or Hughes, Liu and Caughy [1978]), but exactly preserve other fundamental integrals of motion such as global angular momentum (see Marsden [1988] and references therein).

\section{Covariant Three-Dimensional Elasticity}

We summarize the notation to be used in the description of three dimensional elastodynamics, following to a large extent the usage of Marsden and Hughes [1983]. Emphasis is placed on a covariant formulation of the field equations independent of the choice of coordinate charts.

\section{The Configuration Space}

Let $(\mathcal{B}, \mathbf{G})$ and $(\mathcal{Y}, \mathbf{g})$ be two smooth Riemannian manifolds carrying metrics $\mathbf{G}$ and $\mathbf{g}$ respectively. Typically we have $\mathcal{B} \subset \mathcal{Y}$, where $\mathcal{Y}=\mathbb{R}^{3}$ is the Euclidean three-space with the standard Euclidean metric. We refer to $\mathcal{B}$ as the reference configuration with points denoted by $X \in \mathcal{B}$, and we speak of $\mathcal{Y}$ as the ambient space in which the body $\mathcal{B}$ moves. Points in $\mathcal{Y}$ are denoted $x \in \mathcal{Y}$. We shall consider coordinate charts $\hat{X}^{A}: \mathcal{B} \rightarrow \mathbb{R}$ and $\hat{x}^{a}: \mathcal{Y} \rightarrow \mathbb{R}$ so that the local coordinates of the points $X$ and $x$ are denoted by

$$
X^{A}=\hat{X}^{A}(X) \text { for } X \in \mathcal{B} \text { and } x^{a}=\hat{X}^{a}(x) \text { for } x \in \mathcal{Y} .
$$

The configuration space $\mathcal{C}$ is the set of (orientation-preserving) embeddings $\varphi: \mathcal{B} \rightarrow \mathcal{Y}$; we write

$$
\mathcal{C}=\operatorname{Emb}(\mathcal{B}, \mathcal{Y})
$$

and call the set $\varphi(\mathcal{B})$ the current configuration. It is known that, when suitably topologized, $\mathcal{C}$ is a smooth infinite-dimensional manifold (see Abraham, Marsden and Ratiu [1983], Ebin and Marsden [1970] and references therein).

To construct the tangent space to $\mathcal{C}$ at a configuration $\varphi \in \mathcal{C}$, consider a smooth curve $\varepsilon \mapsto \varphi_{\epsilon}$ such that $\varphi_{\epsilon \mid \epsilon=0}=\varphi$, By definition, $d \varphi_{\epsilon} /\left.d \epsilon\right|_{\epsilon=0}$ is tangent to $\mathcal{C}$ at $\varphi$. Let $X \in \mathcal{B}$; then

$$
\left.\frac{d}{d \epsilon}\right|_{\epsilon=0} \varphi_{\epsilon}(X) \in T_{\varphi(X)} \mathcal{Y}
$$


where $T_{\varphi(X)} \mathcal{Y}$ is the tangent space to $\mathcal{Y}$ at $\varphi(X)$. Consequently, the map

$$
\left.X \in \mathcal{B} \mapsto \frac{d}{d \varepsilon}\right|_{\varepsilon=0} \varphi_{\varepsilon}(X) \in T_{\varphi(X)} \mathcal{Y}
$$

is a vector field over $\varphi: \mathcal{B} \rightarrow \mathcal{Y}$. Hence, we define the tangent space $T_{\varphi} \mathcal{C}$ as

$$
T_{\varphi} \mathcal{C}=\left\{\mathbf{V}_{\varphi}: \mathcal{B} \rightarrow T \mathcal{Y} \mid \mathbf{V}_{\varphi}(X) \in T_{\varphi(X)} \mathcal{Y} \text { for all } X \in \mathcal{B}\right\}
$$

In local coordinates relative to the chart $\left\{X^{A}\right\}$ we have

$$
\mathbf{V}_{\varphi}(X)=V^{i}(X) \frac{\partial}{\partial x^{i}} \in \mathbf{T}_{\varphi(X)} \mathcal{Y} .
$$

We often use the notation $x=\varphi(X)$.

\section{Kinematics}

A motion is a curve of configurations; we let $\varphi_{t}$ be the configuration at time $t$ and write $\varphi_{t}(X)=$ $\varphi(X, t)$. Given a motion $\varphi_{t}$, we define the following quantities:

(i) material velocity: $\mathbf{V}_{t} \in \mathbf{T}_{\varphi_{t}} \mathcal{C}$ given by

$$
\mathbf{V}_{t}(X):=\frac{\partial}{\partial t} \varphi(X, t)
$$

(ii) spatial velocity: $\mathbf{v}_{t} \in \mathfrak{X}\left(\varphi_{t}(\mathcal{B})\right)$ [the space of vector fields on $\varphi_{t}(\mathcal{B})$ ] is defined by

$$
\mathbf{v}_{t}=\mathbf{V}_{t} \circ \varphi_{t}^{-1}
$$

(iii) convective velocity: $\mathcal{V}_{t} \in \mathfrak{X}(\mathcal{B})$ is defined by

$$
\mathcal{V}_{t}=\varphi_{t}^{*}\left(\mathbf{v}_{t}\right):=\mathbf{T} \varphi_{t}^{-1} \circ \mathbf{v}_{t} \circ \varphi_{t}=\mathbf{T} \varphi_{t}^{-1} \circ \mathbf{V}_{t}
$$

The deformation gradient, denoted by $\mathbf{F}_{t}$, is defined to be the tangent map of $\varphi_{t}$; we write $\mathbf{F}_{t}=\mathbf{T} \varphi_{t}$. In coordinates,

$$
\mathbf{F}_{t}=F_{A}^{a} \frac{\partial}{\partial x^{a}} \otimes \mathbf{d} X^{A} ; \quad F_{A}^{a}=\frac{\partial \varphi^{a}}{\partial X^{A}},
$$

where $\mathbf{d} X^{A}$ is the basis dual to $\left(\partial / \partial X^{A}\right)$.

The following diagram illustrates these concepts.

Figure 2.1:

Proposition 2.1 The convected velocity is the negative of the spatial velocity of the inverse motion $\varphi_{t}^{-1}: \mathcal{Y} \rightarrow \mathcal{B}$; i.e.

$$
\mathcal{V}_{t}=-\frac{\partial \varphi_{t}^{-1}}{\partial t} \circ \varphi_{t}
$$

Proof Applying the chain rule to the identity $X=\varphi^{-1}(\varphi(X, t), t)$ gives

$$
\frac{\partial \varphi_{t}^{-1}}{\partial t} \circ \varphi_{t}+T \varphi_{t}^{-1} \circ \mathbf{V}_{t}=\mathbf{0}
$$

and so the result follows by noting that $\mathcal{V}_{t}=T \varphi_{t}^{-1} \circ \mathbf{v}_{\mathbf{t}} \circ \varphi_{t}$. 


\section{The Metric and Convected Metric Tensors. Convected Lie Derivative}

We define the convected metric tensor by the pull-back relation:

$$
\mathbf{C}_{t}=\varphi_{t}^{*}(\mathbf{g}) ; \quad \text { i.e., } \quad C_{A B}=F_{A}^{a} F_{B}^{b} g_{a b} \circ \varphi_{t} .
$$

$\mathbf{C}_{t}$ is called the right Cauchy-Green tensor.

Let $\nabla$ be the Levi-Civita connection associated with the spatial Riemannian metric g. The corresponding Christoffel symbols are given by the standard relation:

$$
\gamma_{a b}^{d}=\frac{1}{2} g^{d c}\left[\frac{\partial g_{a c}}{\partial x^{b}}+\frac{\partial g_{b c}}{\partial x^{a}}-\frac{\partial g_{a b}}{\partial x^{c}}\right] .
$$

We define a Riemannian connection $\tilde{\nabla}$ associated with the convected metric tensor $\mathbf{C}$ by the pullback relation:

$$
\tilde{\nabla}_{\mathcal{W}} \mathcal{V}:=\varphi^{*}\left[\nabla_{\varphi_{*}(\mathcal{W})} \varphi_{*}(\mathcal{V})\right]
$$

for any convected vector fields $\mathcal{V}$ and $\mathcal{W} \in \mathfrak{X}(\mathcal{B})$.

Using the properties of pull-backs and covariant differentiation, we find from (2.14) that the coordinate expression for $\tilde{\nabla}$ is

$$
\Gamma_{A B}^{C}:=\frac{\partial^{2} \varphi^{a}}{\partial X^{A} \partial X^{B}}\left(F^{-1}\right)_{a}^{C}+\left(F^{-1}\right)_{c}^{C} F_{B}^{b} F_{A}^{a} \gamma_{a b}^{c}
$$

It can be readily shown that the connection $\tilde{\nabla}$ is the Levi-Civita connection for $\mathbf{C}$ with the Christoffel symbols given by the standard formula:

$$
\Gamma_{A B}^{D}=\frac{1}{2} C^{D C}\left[\frac{\partial C_{A C}}{\partial X^{B}}+\frac{\partial C_{B C}}{\partial X^{A}}-\frac{\partial C_{A B}}{\partial X^{C}}\right] .
$$

Let $\mathcal{W} \in \mathfrak{X}(\mathcal{B})$ be a convected vector field. We define the convected Lie derivative of $\mathcal{W}$, denoted by $\mathcal{L}_{\mathcal{V}} \mathcal{W}$, as the Lie derivative relative to the convected velocity field $\mathcal{V}_{t}$. Consequently $\mathcal{L}_{\mathcal{V}} \mathcal{W}$ is given by the pull-back relation:

$$
\mathcal{L}_{\mathcal{V}} \mathcal{W}:=\varphi_{t}^{*}\left[\mathcal{L}_{\mathcal{V}_{t}}\left(\varphi_{*} \mathcal{W}\right)\right]
$$

Here $\mathcal{L}_{\mathcal{V}} \mathbf{w}$ is the (spatial) Lie derivative of vector fields $\mathbf{w} \in \mathfrak{X}(\varphi(\mathcal{B})$ ) defined by the formula (see Marsden and Hughes [1983], §1.6)

$$
\mathcal{L}_{v_{t}} \mathbf{w}:=\varphi_{t_{*}} \frac{\partial}{\partial t} \varphi_{t}^{*} \mathbf{w}
$$

\section{Acceleration Vector Fields}

We define the material acceleration $\mathbf{A}_{t}: \mathcal{B} \rightarrow T \mathcal{S}$ and the spatial acceleration $\mathbf{a}_{t}: \mathcal{S} \rightarrow T \mathcal{S}$ associated with the motion $\varphi_{t}$ by the expressions

$$
\mathbf{A}_{t}=\partial^{2} \varphi_{t} / \partial t^{2}=\partial \mathbf{V}_{t} / \partial t, \quad \mathbf{a}_{t}=\mathbf{A}_{t} \circ \varphi_{t}^{-1} .
$$

The convected acceleration $\mathcal{A}_{t}: \mathcal{B} \rightarrow T \mathcal{B}$ is defined by the pull-back relation

$$
\mathcal{A}_{t}=\varphi_{t}^{*}\left(\mathbf{a}_{t}\right)
$$

Proposition 2.2 The convected velocity and acceleration are related by the formula

$$
\mathcal{A}_{t}=\frac{\partial \mathcal{V}_{t}}{\partial t}+\tilde{\nabla}_{\mathcal{V}_{t}} \mathcal{V}_{t}
$$

In coordinates, $\mathcal{A}$ is given by

$$
\mathcal{A}^{A}=\partial \mathcal{V}^{A} \mid \partial t+\mathcal{V}^{C} \mathcal{V}_{, C}^{A}+\Gamma_{C D}^{A} \mathcal{V}^{C} \mathcal{V}^{D}
$$


Proof Recall (Marsden and Hughes [1983] p. 33) that the spatial velocity and accelerations are related by

$$
\mathbf{a}_{t}=\frac{\partial \mathbf{v}_{t}}{\partial t}+\nabla \mathbf{v}_{t} \mathbf{v}_{t}
$$

Now pull back $\nabla_{\mathcal{V}_{t}} \mathbf{V}_{t}$ by $\varphi_{t}$ to get

$$
\varphi_{t}^{*}\left(\nabla \mathbf{v}_{t} \mathbf{v}_{t}\right)=\tilde{\nabla}_{\varphi_{t}^{*} v_{t}} \varphi_{t}^{*} \mathbf{v}_{t}=\tilde{\nabla} \mathcal{V} \mathcal{V}_{t}
$$

The Lie derivative formula (2.18) then gives

$$
\frac{\partial \mathcal{V}_{t}}{\partial t}=\frac{\partial}{\partial t}\left(\varphi_{t}^{*} \mathbf{v}_{t}\right)=\varphi_{t}^{*}\left(\mathbf{L}_{\mathbf{v}_{t}} \mathbf{v}_{t}\right)+\varphi_{t}^{*}\left(\frac{\partial \mathbf{v}_{t}}{\partial t}\right)=\varphi_{t}^{*}\left(\frac{\partial \mathbf{v}_{t}}{\partial t}\right) .
$$

Adding (??) and (??) gives (??).

Next, we record some formulae useful for our subsequent development of the convected equations of motion. Let $T^{*} \mathcal{B}$ be the cotangent bundle of $\mathcal{B}$, and denote by ${ }^{b}: T \mathcal{B} \rightarrow T^{*} \mathcal{B}$ the standard index-lowering action (see Abraham, Marsden and Ratiu [1983]) induced by the convected metric C. Given any vector field $\mathcal{W} \in \mathfrak{X}(\mathcal{B})$ there is a unique one-form $\mathcal{W}^{b}$ defined by the relation

$$
\mathcal{W}_{A}(X) \mathcal{U}^{A}(X)=C_{A B}(X) \mathcal{W}^{A}(X) \mathcal{U}^{B}(X),
$$

for any $\mathcal{U} \in \mathfrak{X}(\mathcal{B})$ and $X \in \mathcal{B}$; consequently, in coordinates, $\mathcal{W}^{b}$ is defined as

$$
\mathcal{W}^{\mathrm{b}}(X):=C_{A B}(X) \mathcal{W}^{B}(X) \mathbf{d} X^{A} .
$$

Similarly, following standard notation, we denote by $\sharp: T^{*} \mathcal{B} \rightarrow T \mathcal{B}$ the index-raising action induced by $\mathbf{C}$. Associated with any $\mathcal{M}: \mathcal{B} \rightarrow T^{*} \mathcal{B}$ there is a unique vector field $\mathcal{M}^{\sharp} \in \mathfrak{X}(\mathcal{B})$, with coordinate expression

$$
\mathcal{M}^{\sharp}(X)=C^{A B}(X) \mathcal{M}_{B}(X) \frac{\partial}{\partial X^{A}},
$$

relative to a chart $\left\{X^{A}\right\}$ on $\mathcal{B}$. With this notation at hand we have

Lemma 2.3 For the convected velocity field, the following relations hold:

$$
\text { i. } \quad \mathcal{L}_{\mathcal{V}} \mathcal{V}^{b}=\frac{1}{2} \mathbf{d}|\mathcal{V}|_{C}^{2}+\left(\tilde{\nabla}_{\mathcal{V}} \mathcal{V}\right)^{b},
$$

which has the coordinate form

$$
\begin{array}{r}
\left(\mathcal{L}_{\mathcal{V}} \mathcal{V}^{b}\right)_{A}=\frac{1}{2} \frac{\partial}{\partial X^{A}}\left[\mathcal{V}^{B} C_{B C} \mathcal{V}^{C}\right]+\mathcal{V}_{A \mid B} \mathcal{V}^{B} . \\
\text { ii. } \quad \dot{\mathbf{C}}=\mathcal{L}_{\mathcal{V}} \mathbf{C} ; \quad \text { i.e., }, \quad \dot{C}_{A B}=\mathcal{V}_{A \mid B}+\mathcal{V}_{B \mid A} .
\end{array}
$$

Proof In a coordinate chart $\left\{X^{A}\right\}$ we have:

$$
\begin{aligned}
\left(\mathcal{L}_{\mathcal{V}} \mathcal{V}^{b}\right)_{A} & =\mathcal{V}_{A \mid B} \mathcal{V}^{B}+\left.\mathcal{V}_{B} \mathcal{V}^{B}\right|_{A} \\
& =\frac{1}{2} \frac{\partial}{\partial X^{A}}\left[\mathcal{V}_{B} \mathcal{V}^{B}\right]+\mathcal{V}_{A \mid B} \mathcal{V}^{B} \\
& =\frac{1}{2} \frac{\partial}{\partial X^{A}}\left[\mathcal{V}^{B} C_{B C} \mathcal{V}^{C}\right]+\mathcal{V}_{A \mid B} \mathcal{V}^{B}
\end{aligned}
$$

where a vertical bar denotes covariant differentiation relative to the convected connection $\nabla$. This proves formula i. Since the Lie derivative is natural with respect to pull-backs, from (2.18) we have

$$
\mathcal{L}_{\mathcal{V}_{t}} \mathbf{C}_{t}=\varphi_{t}^{*} \mathcal{L}_{\mathbf{V}_{t}}\left(\varphi_{t_{*}} \mathbf{C}_{t}\right)=\varphi_{t}^{*} \mathcal{L}_{\mathbf{V}_{t}} \mathbf{g}=2 \varphi_{t}^{*} \mathbf{d}
$$


where $\mathbf{d}:=\left[v_{a \mid b}+v_{b \mid a}\right] \mathbf{d} x^{a} \otimes \mathbf{d} x^{b}$ is the (spatial) rate of deformation tensor. Formula ii. then follows by virtue of the well known relation $\dot{\mathbf{C}}=2 \varphi_{t}^{*}(\mathbf{d})$.

In view of formula ii. one says that $\mathbf{C}$ is Lie-dragged by the flow generated by the convected velocity field.

\section{The Convected Volume Element and One-Form Densities}

Next, we turn our attention to the definition of volume elements. Let $d^{3} X:=d X^{1} \wedge d X^{2} \wedge d X^{3}$ and $d^{3} x:=d x^{1} \wedge d x^{2} \wedge d x^{3}$ be the standard coordinate volume elements in $\mathcal{B}$ and $\mathcal{S}$, respectively, and let $\Lambda_{x}^{3}(\mathcal{S})$ and $\Lambda_{X}^{3}(\mathcal{B})$ denote the space of 3 -forms at $x \in \mathcal{S}$ and $X \in \mathcal{B}$, so that $d^{3} X \in \Lambda_{X}^{3}(\mathcal{B})$ and $d^{3} x \in \Lambda_{X}^{3}(\mathcal{S})$. Associated with $\mathbf{g}$ and $\mathbf{G}$ are unique volume densities, denoted by $\mu(\mathbf{g})$ and $\mu(\mathbf{G})$, respectively, and defined locally as

$$
\mu(\mathbf{g}):=\{\operatorname{det}[\mathbf{g}]\}^{1 / 2} d^{3} x, \quad \mu(\mathbf{G}):=\{\operatorname{det}[\mathbf{G}]\}^{1 / 2} d^{3} X .
$$

We use notations $\mu(\mathbf{G}) \in\left|\Lambda^{3}(\mathcal{B})\right|$ and $\mu(\mathbf{g}) \in\left|\Lambda^{3}(\mathcal{S})\right|$ to designate volume densities.

The change of variables formula yields the local representation $d^{3} x=\operatorname{det}[\mathbf{F}] d^{3} X$. Since the Jacobian $J_{\varphi}$ is defined as

$$
\mu(\mathbf{g}) \circ \varphi=: J_{\varphi} \mu(\mathbf{G})
$$

we obtain the expression

$$
J_{\varphi}:=\operatorname{det}[\mathbf{F}]\{\operatorname{det}[\mathbf{g}] / \operatorname{det}[\mathbf{G}]\}^{1 / 2} .
$$

In the convected description, the $\mathbf{C}$-volume density associated with the metric $\mathbf{C}$ is locally defined as

$$
\mu(\mathbf{G}):=\{\operatorname{det}[\mathbf{G}]\}^{1 / 2} d^{3} X .
$$

Since $\operatorname{det}[\mathbf{C}]=\operatorname{det}^{2}[\mathbf{F}] \operatorname{det}[\mathbf{g}]$, we obtain

$$
\mu(\mathbf{C}):=J_{\varphi} \mu(\mathbf{G})
$$

from (2.35), (2.36) and (2.37). A one-form convected density is a mapping $\overline{\mathcal{M}}: \mathcal{B} \rightarrow T^{*} \mathcal{B} \otimes \Lambda^{3}(\mathcal{B}) \mid$ obtained as the tensor product of a convected one-form $\mathcal{M}: \mathcal{B} \rightarrow T^{*} \mathcal{B}$ with convected volume element $\mu(C)$. Accordingly, we have

$$
\overline{\mathcal{M}}=\mathcal{M} \otimes \mu(\mathbf{C})=J_{\varphi} \mathcal{M} \otimes \mu(\mathbf{G}) .
$$

Next, we define the divergence operator, which is needed in the formulation of the equations of motion. By recalling the covariant definition of the divergence (see e.g., Abraham, Marsden, and Ratiu [1983] page 389), we have the following expressions in the convected and spatial descriptions:

$$
\left(\operatorname{Div}_{\mathbf{C}} \mathcal{W}\right) \mu(\mathbf{C}):=\mathcal{L}_{\mathcal{W}_{t}} \mu(\mathbf{C}) ; \quad(\operatorname{div} \mathbf{g} \mathbf{w}) \mu(\mathbf{g})=\mathcal{L}_{\mathcal{W}_{t}} \mu(\mathbf{g}),
$$

for all vector fields $\mathcal{W} \in \mathfrak{X}(\mathcal{B})$ and $\mathbf{w} \in \mathfrak{X}(\varphi(\mathcal{B}))$. Vector fields, volume force densities and Lie derivatives are related in a simple manner. For the convected vector field density, one has the useful formula:

$$
\mathcal{L}_{\mathcal{V}} \overline{\mathcal{W}}=\mathcal{L}_{\mathcal{V}} \mathcal{W} \otimes \mu(\mathbf{C})+\left(\operatorname{Div}_{\mathbf{C}} \mathcal{V}\right) \otimes \mathcal{W} \mu(\mathbf{C})
$$

which follows at once from (2.40) and standard properties of the Lie derivative. 


\section{The Stress Tensor and Covariance}

We assume the existence of a stored energy function $W: \mathcal{M}_{\mathcal{S}} \times \mathcal{C} \times \mathcal{M}_{\mathcal{B}} \rightarrow \mathbb{R}$, where $\mathcal{M}_{\mathcal{S}}$ is the space of Riemannian metrics on $\mathcal{S}$, and $\mathcal{M}_{\mathcal{B}}$ is the space of Riemannian metrics on $\mathcal{B}$, of the form

$$
W=\bar{W}(\mathbf{g}, \mathbf{F}, \mathbf{G}),
$$

where $\bar{W}$ depends only on the point values of $\mathbf{g}, \mathbf{F}$, and $\mathbf{G}$. This dependence is in keeping with the classical assumption that the stored energy function in an elastic material depends on the configuration $\varphi$ only locally through the point values of the deformation gradient $\mathbf{F}$ (see Marsden and Hughes [1983], $\S \S 3.2$ and 3.3). The dependence of the stored energy $\bar{W}$ on the metric tensor $\mathrm{g}$ is essential to introduce the notion of covariance which embodies objectivity (or material frameindifference) as a particular case. Covariance is the statement of (left) invariance to the group of spatial diffeomorphisms, whereas objectivity merely requires (left) invariance relative to the group of spatial isometries. Clearly, the former notion implies the latter, but not conversely. We recall that relativistic elasticity is a covariant theory.

To introduce the notion of covariance we begin by summarizing a few facts concerning the actions of the groups of spatial and material diffeomorphism on the configuration space $\mathcal{C}=\operatorname{Emb}(\mathcal{B}, \mathcal{S})$ for elasticity. For further details, including functional analysis issues not addressed here, we refer to Ebin and Marsden [1973].

i. The groups of spatial and material diffeomorphisms. We denote by $\operatorname{Diff}(\mathcal{B})$ and $\operatorname{Diff}(\mathcal{S})$ the groups of diffeomorphisms in $\mathcal{B}$ and $\mathcal{S}$, respectively. Let $\varphi \in \mathcal{C}$. Define the left and right translations of $\varphi \in \mathcal{C}$ as the mappings

$$
L_{\eta}: \operatorname{Diff}(\mathcal{S}) \times \mathcal{C} \rightarrow \mathcal{C}, \quad R_{\eta}: \mathcal{C} \times \operatorname{Diff}(\mathcal{B}) \rightarrow \mathcal{C},
$$

given by

$$
(\eta, \varphi) \mapsto L_{\eta}(\varphi):=\eta \circ \varphi,(\varphi, \eta) \mapsto R_{\eta}(\varphi):=\varphi \circ \eta .
$$

The tangent maps associated with these actions are obtained in the standard manner as follows. Consider a smooth curve $\varepsilon \mapsto \varphi_{\varepsilon} \in \mathcal{C}$ such that $\left.\varphi_{\varepsilon}\right|_{\varepsilon=0}=\varphi$, and $d \varphi_{\varepsilon} /\left.d \varepsilon\right|_{\varepsilon=0}=\mathbf{V}_{\varphi}$. Then, the tangent maps to $L_{\eta}$ and $R_{\eta}$ are computed as

$$
\left(T_{\varphi} L_{\eta}\right)\left(\mathbf{V}_{\varphi}(X)\right)=\left.\frac{d}{d \varepsilon}\right|_{\varepsilon=0} L_{\eta} \varphi_{\varepsilon}(X)=\left.\frac{d}{d \varepsilon}\right|_{\varepsilon=0} \eta\left(\varphi_{\varepsilon}(X)\right)=D \eta(\varphi(X)) \cdot \mathbf{V}_{\varphi}(X)
$$

Consequently,

$$
T_{\varphi} L_{\eta}\left(\mathbf{V}_{\varphi}\right)=T \eta \circ \mathbf{V}_{\varphi}
$$

for any $\eta \in \operatorname{Diff}(\mathcal{S})$. A similar calculation for $\eta \in \operatorname{Diff}(\mathcal{B})$ shows that

$$
\mathbf{T}_{\varphi} \mathbf{R}_{\eta}\left(\mathbf{V}_{\varphi}\right)=\mathbf{V}_{\varphi \circ \eta^{-1}}
$$

The mechanical interpretation of these relations should be clear. We regard left translations by $\operatorname{Diff}(\mathcal{S})$ as superposed motions, not necessarily rigid, onto the current configuration. By virtue of (2.46), under any superposed $\eta \in \operatorname{Diff}(\mathcal{S})$ material vector fields at $\varphi(X)$ are transformed tensorially to vector fields at $\eta(\varphi(X))$. On the other hand, we regard right translations by $\operatorname{Diff}(\mathcal{B})$ as diffeomorphic changes of reference configuration $(\mathcal{B})$ that, by virtue of $(2.47)$, shift material points $X \in \mathcal{B}$ to $\eta^{0}(X)$, for any $\eta^{0} \in \operatorname{Diff}(\mathcal{B})$.

It can be readily shown that the action of $\operatorname{Diff}(\mathcal{S})$ on spatial vector fields is by push-forward; i.e.:

$$
\eta_{*}(\mathbf{w}) \in \mathfrak{X}(\varphi((\mathcal{B})) \quad \text { for } \quad \mathbf{w} \in \mathfrak{X}(\varphi(\mathcal{B})) .
$$

ii. Covariant stored energy functions. We say that a stored energy function is covariant if it is left-invariant under the action of $\operatorname{Diff}(\mathcal{S})$; accordingly, in view of $(2.46)$ and $(2.48), W(\mathbf{g}, \mathbf{F}, \mathbf{G})$ is covariant if:

$$
\eta \circ \bar{W}(\mathbf{g}, \mathbf{F}, \mathbf{G})=\bar{W}\left(\eta_{*} \mathbf{g}, T \eta \circ \mathbf{F}, \mathbf{G}\right) .
$$


For any $\eta \in \operatorname{Diff}(\mathcal{S})$. The classical result that $W$ depends only on the point values of $\mathbf{C}=\varphi^{*}(\mathbf{g})$ is an immediate consequence of the covariant assumption (2.49). To see this, consider the case $\mathcal{B} \subset \mathcal{S}$, so that $\operatorname{Diff}(\mathcal{B}) \subset \operatorname{Emb}(\mathcal{B}, \mathcal{S})$. For $\eta=\varphi^{-1},(2.49)$ becomes

$$
\varphi^{-1} \circ \bar{W}(\mathbf{g}, \mathbf{F}, \mathbf{G})=\bar{W}\left(\varphi^{*} \mathbf{g}, \mathbf{T} \varphi^{-1} \circ \mathbf{F}, \mathbf{G}\right)=W(\mathbf{C}, \mathbf{1}, \mathbf{G}),
$$

where $\mathbf{1}$ is the identity two-point tensor. Consequently, there is a function $\overline{\bar{W}}$ of the point values of metrics on $\mathbf{B}$ such that

$$
\bar{W}(\mathbf{g}, \mathbf{F}, \mathbf{G})=\overline{\bar{W}}(\mathbf{C}, \mathbf{G}) .
$$

Let $\boldsymbol{\sigma}$ be the Cauchy stress tensor and let $\boldsymbol{\Sigma}$ be the convected stress tensor, which is defined by the pull-back relation

$$
\boldsymbol{\Sigma}:=\varphi^{*}(\boldsymbol{\sigma}) .
$$

Let $\varrho(x, t)$ and $\varrho_{0}(X, t)$ denote the density functions in the current and reference configurations, respectively. Define the convected density function as $\mathcal{R}:=\varphi^{*}(\varrho)=\varrho \circ \varphi$. We then have the following constitutive equations:

$$
\boldsymbol{\sigma}=2 \varrho \frac{\partial \bar{W}(\mathbf{g}, \mathbf{F}, \mathbf{G})}{\partial \mathbf{g}} \text { and } \quad \boldsymbol{\Sigma}=2 \mathcal{R} \frac{\partial \overline{\bar{W}}(\mathbf{C}, \mathbf{G})}{\partial \mathbf{C}},
$$

Relation (2.53) is referred to as the spatial Doyle-Ericksen formula (see Marsden and Hughes [1983] $\S 3.3$, and, for the material counterpart, Simo and Marsden [1984]). In terms of the Lagrangian strain tensor defined by $\mathbf{E}=(\mathbf{C}-\mathbf{G}) / 2$, and the Eulerian strain tensor defined by $\mathbf{e}=\varphi_{*}(\mathbf{E})=\left(\mathbf{g}-\mathbf{b}^{-1}\right) / 2$, where $\mathbf{b}^{-1}=\varphi_{*}(\mathbf{G})$ is the Finger deformation tensor, formulae (2.53) read

$$
\boldsymbol{\sigma}=\varrho \frac{\partial \bar{W}(\mathbf{e}, \mathbf{F}, \mathbf{G})}{\partial \mathbf{e}} \text { and } \quad \boldsymbol{\Sigma}=\mathcal{R} \frac{\partial \overline{\bar{W}}(\mathbf{E}, \mathbf{G})}{\partial \mathbf{E}} .
$$

Note that the dependence of $\overline{\bar{W}}$ on the material metric tensor $\mathbf{G}$ has been explicitly assumed in equation (2.51), but that $\mathbf{G}$ is treated as a parameter as far as the covariance assumption is concerned.

Next, we consider the invariance group of $W$ on the right. According to (2.47), $\operatorname{Diff}(\mathcal{B})$ acts on the right on vector fields $\mathbf{V}_{\varphi}$ by shifting the base points. On the other hand, it can be readily seen that the action of $\operatorname{Diff}(\mathcal{B})$ on convected vector fields $\mathcal{W} \in \mathfrak{X}(\mathcal{B})$ is by push-forward, i.e., for $\eta^{0} \in \operatorname{Diff}(\mathcal{B})$

$$
\mathcal{W} \in \mathfrak{X}(\mathcal{B}) \mapsto \eta_{*}^{0} \mathcal{W} \in \mathfrak{X}(\mathcal{B}) .
$$

[This is called the adjoint action]. Then, the stored energy function, $\overline{\bar{W}}(\mathbf{C}, \mathbf{G})$ is right-invariant under $\operatorname{Diff}(\mathcal{B})$ if :

$$
\overline{\bar{W}}(\mathbf{C}, \mathbf{G}) \circ \eta^{0}=\overline{\bar{W}}\left(\eta_{*}^{0} \mathbf{C}, \eta_{*}^{0} \mathbf{G}\right),
$$

for any $\eta^{0} \in \operatorname{Diff}(\mathcal{B})$. That right-invariance is equivalent to isotropy follows by considering the case $\mathcal{B} \subset \mathcal{S}$. Choosing $\eta^{0}=\varphi$ we conclude from $(2.56)$ that

$$
\overline{\overline{\mathbf{W}}}(\mathbf{C}, \mathbf{G}) \circ \varphi^{-1}=\overline{\overline{\mathbf{W}}}\left(\mathbf{g}, \mathbf{b}^{-1}\right),
$$

which is the classical expression for the stored energy function of an isotropic material. Thus, whereas left-invariance under Diff $(\mathcal{S})$, i.e., covariance, is a fundamental physical requirement, rightinvariance under Diff $(\mathcal{B})$ merely expresses a particular constitutive behavior; namely isotropy. 


\section{The Hamiltonian}

In the Hamiltonian formalism for the material description, the kinetic and potential energies are expressed in the variables on $T^{*} \mathcal{C}$; i.e., in the variables $\varphi \in \mathcal{C}$, and its conjugate momentum $\mathbf{M}_{\varphi}$.

We start by defining the cotangent space $T_{\varphi}^{*} \mathcal{C}$ at $\varphi \in \mathcal{C}$ as a vector space in duality with $T_{\varphi} \mathcal{C}$ by means of a weakly nondegenerate pairing

$$
\langle\cdot, \cdot\rangle: T_{\varphi}^{*} \mathcal{C} \times T_{\varphi} \mathcal{C} \rightarrow \mathbb{R}
$$

which is constructed as follows. Let $\boldsymbol{\alpha}_{\varphi}=\boldsymbol{\alpha}_{\varphi} d V$ be a one-form density covering $\varphi \in \mathcal{C}$, where $d V=\mu(\mathbf{G})$. As in $(2.39), \overline{\boldsymbol{\alpha}}_{\varphi}$ is given locally as $\left(\boldsymbol{\alpha}_{i}(X) d V_{X}\right) d X^{i}$. The natural pairing between one-form densities $\overline{\boldsymbol{\alpha}}_{\varphi}(X) \in T_{\varphi(x)}^{*} \mathcal{S} \otimes\left|\Lambda_{X}(\mathcal{B})\right|$ at $\varphi(X)$ and vectors $\mathbf{V}_{\varphi}(X) \in T_{\varphi(x)} \mathcal{S}$ is given locally in coordinates as

$$
\left\langle\overline{\boldsymbol{\alpha}}_{\varphi}, \mathbf{V}_{\varphi}\right\rangle:=\int_{B} \alpha_{i}(X) V^{i}(X) \mu(\mathbf{G}),
$$

where $\mathbf{V}_{\varphi}(X)=V^{i}(X)\left(\partial \mid \partial x^{i}\right)$. With this pairing, $T^{*} \mathcal{C}$ becomes

$$
T_{\varphi}^{*} \mathcal{C}:=\left\{\overline{\boldsymbol{\alpha}}_{\varphi}: \mathcal{B} \rightarrow T^{*} \mathcal{S} \otimes\left|\Lambda^{3}(\mathcal{B})\right|\left|\bar{\alpha}_{\varphi}(X) \in T_{\varphi(X)}^{*} \mathcal{S} \otimes\right| \Lambda_{X}^{3}(\mathcal{B}) \mid\right\} .
$$

We consider next the Hamiltonian for elasticity in the material, convected and spatial descriptions.

i. Material description. We derive the appropriate expression by starting with the Hamiltonian relative to $T \mathcal{C}$, which is expressed in terms of configurations $\varphi$ and material velocities $\mathbf{V}_{\varphi}$, is given by

$$
\mathbf{H}\left(\mathbf{g} ; \mathbf{V}_{\varphi}, \mathbf{G}\right):=\int_{\mathcal{B}} \varrho_{0}(X)\left|\mathbf{V}_{\varphi}(X)\right|_{\mathbf{g}}^{2} \mu_{X}(\mathbf{G})+\int_{\mathcal{B}} \varrho_{0}(X) \bar{W}(\mathbf{g}(\varphi(X)), \mathbf{F}(X), \mathbf{G}(X)) \mu_{X}(\mathbf{G})
$$

where $|\cdot|_{g}$ denotes the length induced by the spatial metric g. Observe that the Hamiltonian (2.61) depends parametrically on the spatial metric g; i.e., $H: \mathcal{M}_{\mathcal{S}} \times T \mathcal{C} \times \mathcal{M}_{\mathcal{B}} \rightarrow \mathbb{R}$. We get the material description of (2.61) by transforming it to a function defined on $T^{*} \mathcal{C}$. For this purpose we introduce the weak Riemannian metric on $\mathcal{C}$ defined by the kinetic energy; i.e.:

$$
\left\langle\left\langle\mathbf{V}_{\varphi}, \mathbf{W}_{\varphi}\right\rangle\right\rangle_{X}:=\int_{\mathcal{B}} V^{i}(X) W^{j}(X) g_{i j}(\varphi(X)) \varrho_{0}(X) \mu_{X}(\mathbf{G}) .
$$

In finite dimensions, a metric on a manifold induces a bundle metric on the co-tangent bundle. In infinite dimensions, on the other hand, this need not be the case. In the present situation we give an explicit construction following Marsden, Ratiu and Weinstein [1984b]. Let $\overline{\boldsymbol{\alpha}}_{\varphi}=\boldsymbol{\alpha}_{\varphi} d V$ and $\overline{\mathcal{B}}_{\varphi}=\mathcal{B}_{\varphi} d V \in T^{*} \mathcal{C} \otimes|\Lambda(\mathcal{B})|$, where $d V=\mu(\mathbf{G})$ is the volume element. Then $\overline{\boldsymbol{\alpha}}_{\varphi} /\left(\varrho_{0} d V\right)$ and $\overline{\mathcal{B}}_{\varphi} /\left(\varrho_{0} d V\right)$ are one-forms over $\varphi \in \mathcal{C}$ that when evaluated at $\varphi(X)$ are elements of $T_{\varphi(X)} \mathcal{S}$. Now, $T_{\varphi(X)} \mathcal{S}$ is a finite-dimensional Riemannian manifold with metric $\mathbf{g}(\varphi(X))$. Consequently, we have the standard index-lowering and index-raising actions induced by $\mathbf{g}(\varphi(X))$; explicitly, as in $(2.27)$ and (2.28) for any $\mathbf{V}_{\varphi}(X) \in T_{\varphi(X)} \mathcal{S}$ and $\boldsymbol{\alpha}_{\varphi}(X) \in T_{\varphi(X)}^{*} \mathcal{S}$ we define the associated one-form and vector field by

$$
\mathbf{V}_{\varphi}^{\mathrm{b}}(X)=\mathbf{g}_{i j}(\varphi(X)) V^{j}(X) \mathbf{d} x^{i} ; \quad \boldsymbol{\alpha}_{\varphi}^{\sharp}(X)=g^{i j}(\varphi(X)) \alpha_{j}(X) \frac{\partial}{\partial x^{i}} .
$$

These definitions induce a bundle metric on $T^{*} \mathcal{C}$ by the expression

$$
\left(\overline{\boldsymbol{\alpha}}_{\varphi}, \overline{\mathcal{B}}_{\varphi}:=\left\langle\left\langle\mathbf{V}_{\varphi}, \mathbf{W}_{\varphi}\right\rangle\right\rangle,\right.
$$

where $\mathbf{V}_{\varphi}(X)=\left[\overline{\boldsymbol{\alpha}}_{\varphi}\left(\varrho_{0} d V\right)\right]^{\sharp}=\left[\boldsymbol{\alpha}_{\varphi} / \varrho_{0}\right]^{\sharp}$ and $\left.\mathbf{W}_{\varphi}(X)=\left[\overline{\mathcal{B}}_{\varphi} / \varrho_{0} d V\right)\right]^{\sharp}$. We denote by $\|\cdot\|$ the bundle norm defined by the metric (2.64). 
With this notation, we define the material momentum density as

$$
\overline{\mathbf{M}}_{\varphi}:=\varrho_{0} \mathbf{V}_{\varphi}^{b} \mu(\mathbf{G}) \in T_{\varphi}^{*} \mathcal{C} .
$$

In view of (2.62), (2.64) and (2.66), the kinetic energy term in (2.61) becomes $\left\|\mathbf{M}_{\varphi}\right\| / 2$ and the Hamiltonian takes the form:

$$
H\left(\mathbf{g} ; \varphi, \overline{\mathbf{M}}_{\varphi} ; \mathbf{G}\right)=\frac{1}{2}\left\|\mathbf{M}_{\varphi}\right\|^{2}+\int_{\mathcal{B}} \varrho_{0} \bar{W}\left(\mathbf{g} \cdot \varphi, \mathbf{F}_{t}, \mathbf{G}\right) \mu(\mathbf{G}) .
$$

Again, we observe that $H: \mathcal{M}_{\mathcal{S}} \times T^{*} \mathcal{C} \times \mathcal{M}_{\mathcal{B}} \rightarrow \mathbb{R}$ depends parametrically on the metrics $\mathbf{g}$ and $\mathbf{G}$. In addition, $\mathbf{H}$ is subject to the covariance assumption.

ii Convected description. To express the Hamiltonian in the convected representation we first transform the kinetic energy term using relation (2.9) between convected and material velocity fields. We have, in coordinates,

$$
\begin{aligned}
\left\|\mathbf{M}_{\varphi}\right\|^{2} & =\int_{\mathcal{B}} \varrho_{0}(X) V^{a}(X) V^{b}(X) g_{a b}(\varphi(X)) \mu_{X}(\mathbf{G}) \\
& =\int_{\mathcal{B}} \varrho_{0}(X) F_{A}^{a}(X) F_{B}^{b}(X) g_{a b}(\varphi(X)) \mathcal{V}^{A}(X) \mathcal{V}^{B}(X) \mu_{X}(\mathbf{G}) \\
& =\int_{\mathcal{B}} \varrho_{0}(X) C_{A B}(X) \mathcal{V}^{A}(X) \mathcal{V}^{B}(X) \mu_{X}(\mathbf{G}) .
\end{aligned}
$$

Sometimes, we shall write $\mu_{X}(\mathbf{G})$ as just $\mu(\mathbf{G})$, suppressing the variable $\xi$. In view of the integrand in (2.67), we define the convected one-form momentum density to be $\overline{\mathcal{M}}=\mathcal{M} \otimes \mu(\mathbf{C})$, where $\mathcal{M}$ is a one-form on $\mathcal{B}$, and $\mu(\mathbf{C})$ is the convected volume element defined by $(2.37)$, as

$$
\begin{aligned}
\overline{\mathcal{M}} & :=\left[\varrho_{0}(X) C_{A B}(X) \mathcal{V}^{B}(X)\right] \mathbf{d} X^{A} \otimes \mu(\mathbf{G}) \\
& =\left[\mathcal{R}(X) C_{A B}(X) \mathcal{V}^{B}(X)\right] \mathbf{d} X^{A} \otimes \mu(\mathbf{C}) .
\end{aligned}
$$

(Recall that $\mathcal{R}$ is the convected density). Consequently, we have the equivalent expression

$$
\overline{\mathcal{M}}:=\varrho_{0} \mathcal{V}_{t}^{b} \otimes \mu(\mathbf{G})=\mathcal{R}_{t} \mathcal{V}_{t}^{b} \otimes \mu(\mathbf{C}),
$$

where $^{b}: T \mathcal{B} \rightarrow T^{*}$ denotes the lowering-index action induced by $\mathbf{C}$, as defined by (2.27). Again this construction induces a bundle metric given, locally, by the expression

$$
\langle\overline{\mathcal{M}}, \overline{\mathcal{M}}\rangle:=\int_{\mathcal{B}} \frac{1}{\varrho_{0}}\left(\mathcal{M}^{\sharp}, \mathcal{M}^{\sharp}\right)_{C} \mu(\mathbf{G}),
$$

where

$$
\left(\mathcal{M}^{\sharp}, \mathcal{M}^{\sharp}\right)_{C}:=\left(\varrho_{0}(X) \mathcal{V}^{A}(X)\right)\left(\varrho_{0}(X) \mathcal{V}^{B}(X)\right) C_{A B}(X)
$$

is the local inner product induced by $\mathbf{C}$ on $T_{X} \mathcal{B}$. The Hamiltonian in the convected description then becomes

$$
H(\overline{\mathcal{M}}, \mathbf{C}, \mathbf{G}):=\frac{1}{2}\langle\overline{\mathcal{M}}, \overline{\mathcal{M}}\rangle+\int_{\mathcal{B}} \varrho_{0} W(\mathbf{C}, \mathbf{G}) \mu(\mathbf{G}) .
$$

Note that the kinetic energy is now a function of $\overline{\mathcal{M}}$ and $C$ alone. We also note that the kinetic energy is just one half the squared length of the momentum density in the metric on the space of convective momentum densities that is induced by (2.70). We regard (2.71) as the energy induced on the original phase space of $\varphi, \bar{M}_{\varphi}$, g after factoring by the group of spatial diffeomorphisms $\operatorname{Diff}(\mathcal{S})$. (Again $\mathbf{G}$ enters parametrically.) This idea is central to the reduction procedure explained in the next section. 
iii. Spatial description. The expression for the kinetic energy in the spatial description readily follows by recalling relation (2.8) between material and spatial velocity fields. We have

$$
\begin{aligned}
\frac{1}{2}\left\|\mathbf{M}_{\varphi}\right\|^{2} & =\frac{1}{2} \int_{\mathcal{B}} \varrho_{0}(X) V^{a}(X) V^{b}(X) g_{a b}(\varphi(X)) \mu_{X}(\mathbf{G}) \\
& =\frac{1}{2} \int_{\varphi_{t}(\mathcal{B})} \varrho_{t}(x) v^{q}(x) v^{b}(x) g_{a b}(x) \mu_{x}(\mathbf{g}) .
\end{aligned}
$$

Thus, we define the spatial momentum density $\overline{\mathbf{m}}_{t}$ as

$$
\overline{\mathbf{m}}_{t}:=\varrho v_{t}^{b} \otimes \mu(\mathbf{g})
$$

where $^{b}: T S \rightarrow T^{*} S$ now denotes the lowering-index action induced by the spatial metric $\mathbf{g}$. As in the convected description, this process defines the bundle metric

$$
\langle\overline{\mathbf{m}}, \overline{\mathbf{m}}\rangle:=\int_{\varphi_{t}(\mathcal{B})} \frac{1}{\varrho_{t}(x)} m_{a}(x) m_{b}(b)(x) g^{a b}(x) \mu_{x}(g)
$$

in terms of which, the kinetic energy in the spatial description becomes $\langle\overline{\mathbf{m}}, \overline{\mathbf{m}}\rangle / 2$.

The central issue in the Hamiltonian formalism in the spatial description concerns the appropriate formulation of the potential energy term in such a way that the assumed form of the stored energy function does not preclude anisotropic response. Early attempts, e.g., by Marsden, Ratiu and Weinstein [1984], have been restricted to isotropic response. In the present context we proceed as follows. Let

$$
\mathbf{f}_{t}:=-\mathbf{T} \varphi_{t}^{-1} ; \quad \text { i.e., } \mathbf{f}_{t}(x)=-\frac{\partial\left(\varphi^{-1}\right)^{A}}{\partial x^{a}} \frac{\partial}{\partial X^{A}} \otimes \mathbf{d} x^{a},
$$

be the negative of the inverse deformation gradient. Assume a covariant stored energy function of the form

$$
W=\tilde{w}\left(\mathbf{g}, \mathbf{f}_{t}, \mathbf{G} \circ \varphi^{-1}\right),
$$

where, as in the preceeding descriptions, we regard $\tilde{w}$ as depending parametrically on $\mathbf{G}$. Covariance is now understood to be right invariance relative to the group $\operatorname{Diff}(\mathcal{S})$ :

$$
\tilde{w}\left(\mathbf{g}, \mathbf{f}_{t}, \mathbf{G} \circ \varphi^{-1}\right) \circ \eta=\tilde{w}\left(\eta^{*} \mathbf{g}, \mathbf{f}_{t} \circ \mathbf{T} \eta^{-1}, \mathbf{G} \circ \varphi^{-1} \circ \eta^{-1}\right) .
$$

Before proceedings further we remark on the interpretation of $\mathbf{f}_{t}: \mathbf{T} \mathcal{S} \rightarrow \mathbf{T} \mathcal{B}$. By performing a straightforward coordinate calculation we find

$$
\frac{\partial}{\partial t} f_{a}^{A}+\frac{\partial f_{a}^{A}}{\partial x^{b}} v^{b}+f_{b}^{A} \frac{\partial v^{b}}{\partial x^{a}}=0 .
$$

It is apparent that (2.78) is the coordinate expression in a chart $\left\{x^{a}\right\}$ of a one-form on $\mathcal{S}$. This fact motivates the interpretation of $\mathbf{f}_{t}$ as a collection of one-forms: $\boldsymbol{\pi}^{A}: \mathcal{S} \rightarrow T^{*} \mathcal{S}$, (i.e., a vector-valued one form) rather than a two-point tensor:

$$
\mathbf{f}_{t}=\frac{\partial}{\partial X^{A}} \otimes \boldsymbol{\pi}_{t}^{A} ; \quad \boldsymbol{\pi}_{t}^{A}:=f_{a}^{A} \mathbf{d} x^{a} .
$$

Expression (2.78) then reads:

$$
\frac{\partial}{\partial t} \boldsymbol{\pi}_{t}^{A}+\mathcal{L}_{v_{t}} \boldsymbol{\pi}_{t}^{A}=\mathbf{O} .
$$

That is, $\mathbf{f}_{t}$ is Lie-dragged by the flow of the spatial velocity field. By virtue of these observations, the Hamiltonian in the spatial description now reads:

$$
H\left(\mathbf{g}, \overline{\mathbf{m}} ; \mathbf{f}_{t}, \mathbf{G}\right)=\frac{1}{2}\langle\overline{\mathbf{m}}, \overline{\mathbf{m}}\rangle+\int_{\varphi_{t}(\mathcal{B})} \varrho \tilde{w}\left(\mathbf{g}, \mathbf{f}_{t}, G \circ \varphi^{-1}\right) \mu(\mathbf{g}) .
$$




\section{Equations of Motion}

We conclude this section with a summary of the equations of motion in the spatial, material and convected descriptions.

i. Conservation of mass. Let $\varphi_{t} \in \mathcal{C}$ be a motion, $\mathcal{U} \subset \mathcal{B}$ a compact set, and $\varphi_{t}(\mathcal{U}) \subset \mathcal{S}$. Conservation of mass requires that

$$
\int_{\mathcal{U}} \varrho_{0}(X) \mu_{X}(\mathbf{G})=\int_{\varphi_{t}(\mathcal{U})} \varrho_{t}(x) \mu_{x}(\mathbf{g}) .
$$

By the change of variables formula, assuming enough smoothness, we have

$$
\varphi_{t}^{*}\left(\varrho_{t}(x) \mu_{x}(\mathbf{g})\right)=\left(\varrho_{t} \circ \varphi_{t}\right)(X) \varphi^{*}\left(\mu_{x}(g)\right)=\varrho_{0}(X) \mu_{X}(\mathbf{G}) .
$$

Alternatively, since $\mu(\mathbf{g}) \circ \varphi=: J_{\varphi} \mu(\mathbf{G})$ by $(2.35)$, we have the equivalent expression

$$
\varphi_{0}(X)=\left(\varrho_{t} \circ \varphi_{t}\right) \mathbf{J}_{\varphi}(X) .
$$

i.a. Spatial Description. From (2.84) it follows that

$$
\varphi_{t *} \frac{\partial}{\partial t} \varphi_{t}^{*}\left(\varrho_{t} \mu(\mathbf{g})\right)=0
$$

The Lie derivative formula (2.18) then yields

$$
\mathcal{L}_{\mathbf{V}_{t}}\left[\varrho_{t} \otimes \mu(\mathbf{g})\right]=0 .
$$

Alternatively, using the fact that $\mathcal{L}_{\mathbf{V}} \mu(\mathbf{g})=\left(\operatorname{div}_{g} \mathbf{v}\right) \mu(\mathbf{g})$, we recover from (2.87) the classical expression

$$
\frac{\partial \varrho_{t}}{\partial t}+\operatorname{div}_{g}\left(\varrho_{t} \mathbf{v}_{t}\right)=0
$$

Note, however, that either expression (2.84) or expression (2.87) is more convenient than the classical relation (2.88). This observation is crucial in our subsequent development of the Poisson bracket in the convective representation.

i.b. Convected Description. By taking the pull-back of (2.84) and using standard properties of the Lie derivative we obtain

$$
0=\varphi_{t}^{*}\left[\mathcal{L}_{\mathbf{V}_{t}}\left(\varrho_{t} \otimes \mu(\mathbf{g})\right)\right]=\mathcal{L}_{\varphi_{t}^{*} \mathbf{v}_{t}}\left(\varphi_{t}^{*} \varrho_{t} \otimes \varphi_{t}^{*} \mu(\mathbf{g})\right) .
$$

Using (2.15), (2.35) and (2.38) we obtain

$$
\mathcal{L}_{\mathcal{V}_{t}}\left(\mathcal{R}_{t} \otimes \mu(\mathbf{C})\right) \equiv \mathcal{L}_{\mathcal{V}_{\mathbf{t}}}\left(\varrho_{0} \otimes \mu(\mathbf{G})\right)=0 .
$$

Equivalently, by exploiting the connection between Lie derivatives and volume elements we have

$$
\frac{\partial \mathcal{R}_{t}}{\partial t}+\operatorname{Div}_{\mathbf{C}}\left(\mathcal{R}_{t} \mathcal{V}_{t}\right)=0
$$

i.c. Material Description. From either (2.84) or (2.90) we obtain:

$$
\frac{\partial \varrho_{0}}{\partial t}=0
$$

which constitutes the statement of conversation of mass in the material description. 
ii. Conservation of Momentum. We summarize below the covariant version of the classical forms of the local momentum equations in the spatial, convected and material descriptions.

ii.a Spatial Description. Assuming for simplicity zero body forces, we have the classical equations

$$
\operatorname{div} \mathbf{g} \boldsymbol{\sigma}=\varrho_{t}\left[\frac{\partial \mathbf{v}_{t}}{\partial t}+\nabla_{v_{t}} \mathbf{v}_{t}\right] .
$$

To express (2.93) in covariant form; i.e., in a form independent of the choice of coordinates, and in terms of the spatial momentum density, we proceed as follows. The time-differentiation of definition (2.74) for $\overline{\mathbf{m}}_{t}$ and the use of (2.93) yields

$$
\frac{\partial \overline{\mathbf{m}}_{t}}{\partial t}=\frac{\partial \varrho_{t}}{\partial t} \mathbf{v}_{t}^{b} \otimes \mu(\mathbf{g})-\left[\varrho_{t}\left(\nabla_{v_{t}} \mathbf{v}_{t}\right)^{b}-(\operatorname{div} \mathbf{g} \boldsymbol{\sigma})^{b}\right] \otimes \mu(\mathbf{g}) .
$$

On the other hand, by the Lie derivative formula:

$$
\mathcal{L}_{v_{t}} \overline{\mathbf{m}}_{t}=\varrho_{t}\left(\mathcal{L}_{v_{t}} \mathbf{v}_{t}^{b}\right) \otimes \mu(\mathbf{g})+\left(\mathbf{d} \varrho_{t} \cdot \mathbf{v}_{t}\right) \mathbf{v}_{t}^{b} \otimes \mu(\mathbf{g})+\varrho_{t} \mathbf{v}_{t}^{b} \otimes \mathcal{L}_{v_{t}} \mu(\mathbf{g}) .
$$

Again using the fact that $\mathcal{L}_{\mathbf{V}}(\mu(\mathbf{g}))=\left[\operatorname{div}_{g} \mathbf{v}\right] \mu(\mathbf{g})$ along with the spatial counterpart of $(2.31)$, we convert (2.95) to

$$
\mathcal{L}_{v_{t}} \overline{\mathbf{m}}_{t}=\left[\varrho_{t}\left(\nabla_{v_{t}} \mathbf{v}_{t}\right)^{b}+\frac{1}{2} \varrho_{t} \mathbf{d}\left|\mathbf{v}_{t}\right|_{g}^{2}+\left(\mathbf{d} \varrho_{t} \cdot \mathbf{v}_{t}\right) \mathbf{v}_{t}^{b}+\varrho_{t}\left(\operatorname{div}_{g} \mathbf{v}_{t}\right) \mathbf{v}_{t}^{b}\right] \otimes \mu(\mathbf{g}) .
$$

Adding (2.94) and (2.96) and using the continuity equation (2.88) we finally obtain

$$
\frac{\partial \overline{\mathbf{m}}_{t}}{\partial t}+\mathcal{L}_{v_{t}} \overline{\mathbf{m}}_{t}=\left[\left(\operatorname{div}_{g} \boldsymbol{\sigma}\right)^{b}+\frac{\varrho_{t}}{2} \mathbf{d}\left|\mathbf{v}_{t}\right|_{g}^{2}\right] \otimes \mu(\mathbf{g})
$$

which is the desired expression.

ii.b. Convective Expression. A pull-back of the classical balance of momentum equations (2.92) yields

$$
\operatorname{Div}_{\mathbf{C}} \boldsymbol{\Sigma}=\mathcal{R}\left[\frac{\partial \mathcal{V}_{t}}{\partial t}+\tilde{\nabla}_{\mathcal{V}_{t}} \mathcal{V}_{t}\right], \quad \boldsymbol{\Sigma}=\boldsymbol{\Sigma}^{T}
$$

The covariant version of (2.98) may be obtained by the same argument as that leading to (2.97), now phrased in the convective description. Alternatively, since

$$
\frac{\partial \overline{\mathbf{M}}_{t}}{\partial t}=\frac{\partial}{\partial t} \varphi_{t}^{*} \overline{\mathbf{m}}_{t}=\varphi_{t}^{*}\left[\frac{\partial \overline{\mathbf{m}}_{t}}{\partial t}+\mathcal{L}_{v_{t}} \overline{\mathbf{m}}_{t}\right]
$$

by the pull-back of (2.97), one finds

$$
\frac{\partial \overline{\mathbf{M}}_{t}}{\partial t}=\left[\left(\operatorname{Div}_{\mathbf{C}} \boldsymbol{\Sigma}\right)^{b}+\frac{\mathcal{R}_{t}}{2} \mathbf{d}\left|\mathcal{V}_{t}\right|_{\mathbf{C}}^{2}\right] \otimes \mu(\mathbf{C}) .
$$

ii.c. Material Representation. For completeness we also record the form of the momentum equations in the material description. Making use of the Piola transformation (cf., e.g., Marsden and Hughes [1983, Chap. 1]) from the spatial form (2.93) we obtain

$$
\operatorname{DIV}_{\mathbf{G}} \mathbf{P}=\varrho_{0} \frac{\partial \mathbf{V}_{t}}{\partial t} ; \quad \mathbf{P F}_{t}^{T}=\mathbf{F}_{t} \mathbf{P}^{T}
$$

where $\mathbf{P}:=J_{\varphi} \boldsymbol{\sigma} F_{t}^{-1}$ is the (non-symmetric) first Piola-Kirchhoff tensor.

In the next section, we show that the field equations in the convected description are Hamiltonian relative to a non-canonical Lie-Poisson structure on the material phase space reduced on the left by the group of spatial diffeomorphisms. 


\section{The Hamiltonian Structure of Three-Dimensional Elas- ticity in the Material and Convective Representations}

In this section we show that the equations of elastodynamics in the convective representation are Hamiltonian relative to a non-canonical Poisson structure on the space of pairs $(\mathcal{M}, \mathcal{C})$, where $\mathcal{M}$ is the convective momentum density and $\mathbf{C}$ is the Cauchy-Green tensor, as in the preceeding section. This means that the equations of elastodynamics are equivalent to the following condition: For any function $f(\mathcal{M}, \mathbf{C})$,

$$
\dot{f}=\{f, H\},
$$

where $H$ is the Hamiltonian, given by (2.72), and the bracket \{\} appearing in (3.1) satisfies the usual conditions for a Poisson bracket, including Jacobi's identity (see, for example, Marsden and collaborators [1983]).

This bracket is obtained by reducing the canonical bracket on $T^{*} \mathcal{C}$ by the group of spatial isometries of the metric $\mathbf{g}$ on $\mathcal{S}$. Equivalently, as in Marsden, Ratiu and Weinstein [1984a, b], we can incorporate the metrics $\mathbf{g}$ and $\mathbf{G}$ as parameters and then reduce the cotangent bundle $T^{*}\left(\mathcal{M}_{\mathcal{S}} \times \mathcal{C} \times \mathcal{M}_{\mathcal{B}}\right)$ by the left action of $\mathcal{M}_{\mathcal{S}} \times \operatorname{Diff}(\mathcal{S}) \times \mathcal{M}_{\mathcal{B}}$. As is shown in this reference, the result is the same as reducing $\mathcal{M}_{\mathcal{S}} \times T^{*} \mathcal{C} \times \mathcal{M}_{\mathcal{B}}$ by the action of $\operatorname{Diff}(\mathcal{S})$. This reduction procedure will be explicitly explained by direct calculation in what follows. Before reading this section, the reader may find it helpful to review first the parallel case of rigid body dynamics in $\S 4$.

\section{The Canonical Bracket on the Material Phase Space}

We start with the canonical phase space $T^{*} \mathcal{C}$; the space of configurations $\varphi \in \mathcal{C}$ and their canonically conjugate momenta $\overline{\mathbf{M}}_{\varphi}=\mathbf{M}_{\varphi} \otimes \mu(\mathbf{G})$, the material momentum densities, in addition, to accommodate the covariance assumptions and the influence of the reference configuration (i.e., anisotropy), one introduces the spatial and material metrics, $\mathrm{g} \in \mathcal{M}_{\mathcal{S}}$ and $\mathbf{G} \in \mathcal{M}_{\mathcal{B}}$, as additional parameters. Consequently, we consider the material phase space

$$
\mathcal{P}_{\text {can }}:=\mathcal{M}_{\mathcal{S}} \times T^{*} \mathcal{C} \times \mathcal{M}_{\mathcal{B}} .
$$

We write $f\left(\mathbf{g} ; \varphi, \overline{\mathbf{M}}_{\varphi}, \mathbf{G}\right)$ for functions $f: \mathcal{P}_{\text {can }} \rightarrow \mathbb{R}$.

To define the canonical bracket on $\mathcal{P}_{\text {can }}$ we start by introducing the notation of the partial Fréchet and functional derivatives of a function $f: \mathcal{P}_{\text {can }} \rightarrow \mathbb{R}$. The partial Fréchet and functional derivatives of $f: \mathcal{P}_{\text {can }} \rightarrow \mathbb{R}$ relative to $\overline{\mathbf{M}}_{\varphi}$ is defined by considering a curve $\varepsilon \mapsto \overline{\mathbf{M}}_{\varphi}+\varepsilon \delta \overline{\mathbf{M}}_{\varphi}$ and setting

$$
\mathbf{D}_{\overline{\mathbf{M}}_{\varphi}} f \cdot \delta \overline{\mathbf{M}}_{\varphi}:=\left.\frac{d}{d \varepsilon}\right|_{\varepsilon=0} f\left(\mathbf{g}, \varphi, \overline{\mathbf{M}}_{\varphi}+\varepsilon \delta \overline{\mathbf{M}}_{\varphi}, \mathbf{G}\right),
$$

where $\delta \overline{\mathbf{M}}_{\varphi}:=\delta \mathbf{M}_{\varphi} \otimes \mu(\mathbf{G})$ is a one-form density covering $\varphi \in \mathcal{C}$.

The definition of the partial Fréchet derivative of $f: \mathcal{P}_{\text {can }} \rightarrow \mathbb{R}$ with respect to $\varphi \in \mathcal{C}$ requires some caution since $T^{*} \mathcal{C}$ is not simply a product space. Essentially, one needs to "fix" $\overline{\mathbf{M}}_{\varphi}$ while allowing $\varphi \in \mathcal{C}$ to vary. To formalize this intuitive notion, we proceed as in Lewis, Marsden, Montgomery and Ratiu [1987]. We identify $T_{\varphi}^{*} \mathcal{S}$ with $\varphi(\mathcal{B}) \times\left(\mathbb{R}^{3}\right)^{*}$ and denote by $\widetilde{\mathbf{M}}_{\varphi}: \mathcal{B} \rightarrow\left(\mathbb{R}^{3}\right)^{*}$ the principal part of $\mathbf{M}_{\varphi}$; i.e., the projection of $\mathbf{M}_{\varphi}$ onto $\left(\mathbb{R}^{3}\right)^{*}$. Thus we regard $\mathbf{M}_{\varphi}$ as the mapping

$$
\mathbf{M}_{\varphi}=\varphi \times \widetilde{\mathbf{M}}_{\varphi}: \mathcal{B} \rightarrow \varphi(\mathcal{B}) \times\left(\mathbb{R}^{3}\right)^{*} \cong T_{\varphi}^{*} \mathcal{S} .
$$

As usual, we set $\overline{\mathbf{M}}_{\varphi}:=\mathbf{M}_{\varphi} \otimes \mu(\mathbf{G})$.

With these identifications, let $\varepsilon \mapsto \varphi_{\varepsilon} \in \mathcal{C}$ be a smooth curve such that

$$
\delta \varphi:=\left.\frac{d}{d \varepsilon}\right|_{\varepsilon=0} \varphi_{\varepsilon} \in T_{\varphi} \mathcal{C} .
$$


Then, set

$$
\overline{\mathbf{M}}_{\varphi_{\varepsilon}}:=\varphi_{\varepsilon} \times \widetilde{\mathbf{M}}_{\varphi} \otimes \mu(\mathbf{G}),
$$

and define

$$
\mathbf{D}_{\varphi} f \cdot \delta \varphi:=\left.\frac{d}{d \varepsilon}\right|_{\varepsilon=0} f\left(\mathbf{g} ; \varphi_{\varepsilon}, \overline{\mathbf{M}}_{\varphi_{\varepsilon}} ; \mathbf{G}\right) .
$$

Next, we define partial functional derivatives. Since boundary conditions are involved, we consider two possible situations.

i. Pure displacement boundary conditions. The configurations $\varphi \in \mathcal{C}$ are restricted by

$$
\left.\varphi\right|_{\partial \mathcal{B}}=\tilde{\varphi} \quad \text { (prescribed). }
$$

Consequently, the admissible variations $\mathbf{V}_{\varphi} \in T_{\varphi} \mathcal{C}$ must satisfy

$$
\mathbf{V}_{\varphi}(X)=\mathbf{0} \text { for } X \in \partial \mathcal{B}
$$

so that

$$
\overline{\mathbf{M}}_{\varphi}(X)=\mathbf{0} \text { for } X \in \partial \mathcal{B} .
$$

We now define the partial functional derivative, $\delta \bar{f} / \delta \varphi=(\delta f / \delta \varphi) \otimes \mu(\mathbf{G})$, as the one-form density covering $\varphi \in \mathcal{C}$, which is given by the relation

$$
\mathbf{D}_{\varphi} f \cdot \delta \varphi=\int_{\mathcal{B}} \frac{\overline{\delta f}}{\delta \varphi} \cdot \delta \varphi=\int_{\dot{\mathcal{B}}} \frac{\delta f}{\delta \varphi} \cdot \delta \varphi \mu(\mathbf{G})
$$

Similarly, the partial functional derivative $\left(\delta f / \delta \overline{\mathbf{M}}_{\varphi}\right)$, is a vector field covering $\varphi \in \mathcal{C}$ given by

$$
\mathbf{D}_{\overline{\mathbf{M}}_{\varphi}} f \cdot \delta \overline{\mathbf{M}}_{\varphi}=\int_{\mathcal{B}} \frac{\delta f}{\delta \overline{\mathbf{M}}_{\varphi}} \cdot \delta \overline{\mathbf{M}}_{\varphi}=\int_{\mathcal{B}} \frac{\delta f}{\delta \overline{\mathbf{M}}_{\varphi}} \cdot \delta \mathbf{M}_{\varphi} \mu(\mathbf{G}) .
$$

With these definitions, the canonical Poisson bracket $\{\cdot, \cdot\}: \mathcal{F}\left(\mathcal{P}_{\text {can }}\right) \times \mathcal{F}\left(\mathcal{P}_{\text {can }}\right) \rightarrow \mathbb{R}$ takes the form

$$
\{f, g\}=\int_{\mathcal{B}}\left[\frac{\overline{\delta f}}{\delta \varphi} \cdot \frac{\delta g}{\delta \overline{\mathbf{M}}_{\varphi}}-\frac{\overline{\delta g}}{\delta \varphi} \cdot \frac{\delta f}{\delta \overline{\mathbf{M}}_{\varphi}}\right] .
$$

ii. General Boundary conditions. Next we consider general boundary conditions of mixed type. Assuming dead loading, we have

$$
\begin{aligned}
&\left.\varphi\right|_{\partial_{\varphi} \mathcal{B}}=\tilde{\varphi} \text { (prescribed) } \\
&\left.\mathbf{P} \hat{\mathbf{N}}\right|_{\partial_{\sigma} \mathcal{B}}=\tilde{\mathbf{t}} \quad \text { (prescribed) }
\end{aligned}
$$

where $\mathbf{P}$ is the first Piola-Kirchoff tensor, introduced in (2.101), $\hat{\mathbf{N}}$ is the normal to $\partial_{\sigma} \mathcal{B}$, and

$$
\operatorname{closure}\left(\partial_{\varphi} \mathcal{B} \cup \partial_{\sigma} \mathcal{B}\right)=\operatorname{closure}(\partial \mathcal{B}), \quad \partial_{\varphi} \mathcal{B} \cap \partial_{\sigma} \mathcal{B}=\emptyset .
$$

To account for the traction boundary conditions on $\partial_{\sigma} \mathcal{B}$, we modify our definition (3.10) of functional derivative as follows. We set

$$
\mathbf{D}_{\varphi} f \cdot \delta \varphi=\int_{\dot{\mathcal{B}}} \frac{\overline{\Delta f}}{\delta \varphi} \cdot \delta \varphi+\left.\int_{\partial_{\sigma} \mathcal{B}} \frac{\overline{\partial f}}{\delta \varphi} \cdot \delta \varphi\right|_{\partial_{\sigma} \mathcal{B}}
$$

for all variations $\delta \varphi \in \mathbf{T}_{\varphi} \mathcal{C}$ satisfying the essential boundary conditions; i.e.,

$$
\left.\delta \varphi\right|_{\partial_{\varphi} \mathcal{B}}=\mathbf{0} .
$$


Here, $\overline{\Delta f} / \delta \varphi$ is a one-form density defined on points $X \in \partial_{\sigma} \mathcal{B}$. For further elaboration on the possible alternative definitions of partial functional derivatives that account for the (natural) boundary conditions (3.14) we refer to Lewis, Marsden, Montgomery and Ratiu [1986].

We define

$$
\frac{\delta f}{\delta \varphi}:=\frac{\Delta f}{\delta \varphi}+\delta_{\left(\partial_{\sigma} \mathcal{B}\right)} \frac{\partial f}{\delta \varphi} ; \quad \frac{\delta f}{\delta \mathbf{M}_{\varphi}} \equiv \frac{\Delta f}{\delta \mathbf{M}_{\varphi}},
$$

where $\delta_{\left(\partial_{\sigma} \mathcal{B}\right)}$ is the Dirac delta measure on $\mathcal{B}$ concentrated on $\partial_{\sigma} \mathcal{B}$. Then the standard definition (3.12) of the canonical bracket now becomes

$$
\{f, g\}=\int_{\mathcal{B}} \frac{\overline{\Delta f}}{\delta \varphi} \cdot \frac{\delta g}{\delta \overline{\mathbf{M}}_{\varphi}}-\frac{\overline{\Delta g}}{\delta \varphi} \cdot \frac{\delta f}{\delta \overline{\mathbf{M}}_{\varphi}}+\int_{\partial_{\sigma} \mathcal{B}}\left[\left.\frac{\partial f}{\delta \varphi} \cdot \frac{\delta g}{\delta \overline{\mathbf{M}}_{\varphi}}\right|_{\partial_{\sigma} \mathcal{B}}-\left.\frac{\partial g}{\delta \varphi} \cdot \frac{\delta f}{\delta \overline{\mathbf{M}}_{\varphi}}\right|_{\partial_{\sigma} \mathcal{B}}\right] \gamma(\mathbf{G})
$$

where $\overline{\Delta f} / \delta \varphi:=\Delta f / \delta \varphi \otimes \mu(\mathbf{G})$ is a one-form density covering $\varphi: \mathcal{B} \rightarrow \mathcal{S}$, and $\gamma(\mathbf{G})$ is the surface area element on $\partial_{\sigma} \mathcal{B}$ induced by $\mathbf{G}$.

Proposition 3.1 The canonical Hamilton equations $\dot{f}=\{f, H\}$ on material phase space, $\mathcal{P}_{\text {can }}$, yield the material balance laws:

$$
\begin{gathered}
\frac{\partial \varphi}{\partial t}=\mathbf{V}_{t} \quad \text { in } \mathcal{B}, \\
\frac{\partial \overline{\mathbf{M}}_{\varphi}}{\partial t}=\operatorname{DIV}_{\mathbf{G}} \mathbf{P} \otimes \mu(\mathbf{G}) ; \quad \mathbf{P}=\varrho_{0} \frac{\partial W(\mathbf{g} \circ \varphi, T \varphi, \mathbf{G})}{\partial \mathbf{F}} \\
\frac{\partial \tilde{\varphi}}{\partial t}=\mathbf{V}_{t} \quad \text { on } \partial_{\varphi} \mathcal{B}, \quad \text { and } \tilde{\mathbf{t}}=\mathbf{P} \widehat{\mathbf{N}} \quad \text { on } \partial_{\sigma} \mathcal{B} .
\end{gathered}
$$

Proof The Hamiltonian in the material (Lagrangian) description is given by (2.62), i.e.,

$$
\begin{aligned}
H\left(\mathbf{g} ; \varphi, \overline{\mathbf{M}}_{\varphi}, \mathbf{G}\right)= & \frac{1}{2} \int_{\mathcal{B}} \varrho_{0}\left|\mathbf{V}_{t}\right|^{2} \mu(\mathbf{G})+\int_{\mathcal{B}} \varrho_{0} W(\mathbf{g} \circ \varphi, T, \varphi, \mathbf{G}) \mu(\mathbf{G}) \\
& -\int_{\partial_{\sigma} \mathcal{B}} \varphi \cdot \tilde{\mathbf{t}} \gamma(\mathbf{G}),
\end{aligned}
$$

where $\overline{\mathbf{M}}_{\varphi}=\mathbf{M}_{\varphi} \otimes \mu(\mathbf{G})=\varrho_{0} \mathbf{V}_{t}^{b} \otimes \mu(\mathbf{G})$. By considering a curve $\varepsilon \mapsto \varphi_{\varepsilon} \in \mathcal{C}$ with $\left.\varphi_{\varepsilon}\right|_{\varepsilon=0}=\varphi$ and $\left.\left(d \varphi_{\varepsilon} / d \varepsilon\right)\right|_{\varepsilon=0}=\delta \varphi$, and keeping in mind the identifications elaborated upon above, we find

$$
\begin{aligned}
\mathbf{D}_{\varphi} H \cdot \delta \varphi & =\int_{\mathcal{B}} \varrho_{0} \frac{\partial W}{\partial \mathbf{F}}: \operatorname{GRAD} \delta \varphi \otimes \mu(\mathbf{G})-\int_{\partial_{\sigma} \mathcal{B}} \delta \varphi \cdot \tilde{\mathbf{t}} \boldsymbol{\gamma}(\mathbf{G}) \\
& =\int_{\mathcal{B}}-\left[\operatorname{DIV}_{\mathbf{G}} \mathbf{P} \otimes \mu(\mathbf{G})\right] \cdot \delta \varphi+\int_{\partial_{\sigma} \mathcal{B}}(\mathbf{P} \widehat{\mathbf{N}}-\tilde{\mathbf{t}}) \cdot \delta \varphi \boldsymbol{\gamma}(\mathbf{G}) .
\end{aligned}
$$

It follows that

$$
\frac{\overline{\Delta H}}{\delta \varphi}=-\operatorname{DIV}_{\mathbf{G}}[\mathbf{P} \otimes \mu(\mathbf{G})] ; \frac{\overline{\partial H}}{\delta \varphi}=(\mathbf{P} \widehat{\mathbf{N}}-\tilde{\mathbf{t}}) \otimes \gamma(\mathbf{G})
$$

Similarly one finds

$$
\frac{\delta H}{\delta \overline{\mathbf{M}}_{\varphi}}=\mathbf{V}_{t} .
$$

The canonical bracket (3.19) then becomes

$$
\begin{aligned}
\{f, H\}= & \int_{\mathcal{B}} \frac{\overline{\Delta f}}{\delta \varphi} \cdot \mathbf{V}_{\varphi}+\frac{\delta f}{\delta \overline{\mathbf{M}}_{\varphi}} \cdot\left[\operatorname{DIV}_{\mathbf{G}}(\mathbf{P} \otimes \mu(\mathbf{G})]\right. \\
& +\int_{\partial_{\sigma} \mathcal{B}}\left[\left.\frac{\partial f}{\delta \varphi} \cdot \mathbf{V}_{\varphi}\right|_{\partial_{\sigma} \mathcal{B}}+\left.(\tilde{\mathbf{t}}-\mathbf{P} \widehat{\mathbf{N}}) \cdot \frac{\delta f}{\delta \overline{\mathbf{M}}_{\varphi}}\right|_{\partial_{\sigma} \mathcal{B}}\right] \gamma(\mathbf{G}) .
\end{aligned}
$$


On the other hand, for any $f: \mathcal{P}_{\text {can }} \rightarrow \mathbb{R}$ we have

$$
\dot{f}=\int_{\mathcal{B}} \frac{\overline{\Delta f}}{\delta \varphi} \cdot \frac{\partial \varphi}{\partial t}+\frac{\delta f}{\delta \overline{\mathbf{M}}_{\varphi}} \cdot \frac{\partial \overline{\mathbf{M}}_{\varphi}}{\partial t}+\left.\int_{\partial_{\sigma} \mathcal{B}} \frac{\partial f}{\delta \varphi} \cdot \frac{\partial \varphi}{\partial t}\right|_{\partial_{\sigma} \mathcal{B}} \gamma(\mathbf{G})
$$

By comparing (3.25) and (3.26) we obtain the result.

Theorem 3.2 The reduced Poisson bracket on the convective phase space has the following equivalent expressions:

$$
\begin{aligned}
& \{f, g\}=\int_{\mathcal{B}} \overline{\mathcal{M}} \cdot\left[\frac{\delta f}{\delta \overline{\mathcal{M}}}, \frac{\delta g}{\delta \overline{\mathcal{M}}}\right] \\
& +\int_{\mathcal{B}} \mathbf{C}:\left[\frac{\delta f}{\delta \overline{\mathcal{M}}} \otimes \operatorname{Div}_{\mathbf{C}}\left(2 \frac{\delta g}{\delta \mathbf{C}}\right)-\frac{\delta g}{\delta \overline{\mathcal{M}}} \otimes \operatorname{Div}_{\mathbf{C}}\left(2 \frac{\delta f}{\delta \mathbf{C}}\right)\right] \mu(\mathbf{C}) \\
& -\int_{\partial_{\sigma} \mathcal{B}} \mathbf{C}:\left[\frac{\delta f}{\delta \overline{\mathcal{M}}} \otimes 2 \frac{\delta g}{\delta \mathbf{C}} \widehat{\mathbf{N}}-\frac{\delta g}{\delta \overline{\mathcal{M}}} \otimes 2 \frac{\delta f}{\delta \mathbf{C}} \widehat{\mathbf{N}}\right] \boldsymbol{\gamma}(\mathbf{C}) \\
& =\int_{\mathcal{B}} \bar{M} \cdot\left[\frac{\delta f}{\delta \overline{\mathcal{M}}}, \frac{\delta g}{\delta \overline{\mathcal{M}}}\right]+\int_{\mathcal{B}}\left[\frac{\overline{\delta f}}{\delta \mathbf{C}}: \mathcal{L}_{\frac{\delta g}{\delta \overline{\mathcal{M}}}} \mathbf{C}-\frac{\overline{\delta g}}{\delta \mathbf{C}}: \mathcal{L}_{\frac{\delta f}{\delta \overline{\mathcal{M}}}} \mathbf{C}\right] \\
& -\int_{\partial_{\varphi} \mathcal{B}} \mathbf{C}:\left[\frac{\delta g}{\delta \overline{\mathcal{M}}} \otimes 2 \frac{\delta f}{\delta \mathbf{C}} \widehat{\mathbf{N}}-\frac{\delta f}{\delta \overline{\mathcal{M}}} \otimes 2 \frac{\delta g}{\delta \mathbf{C}} \widehat{\mathbf{N}}\right] \boldsymbol{\gamma}(\mathbf{C}) \\
& =\int_{\mathcal{B}} \bar{M} \cdot\left[\frac{\delta f}{\delta \overline{\mathcal{M}}}, \frac{\delta g}{\delta \overline{\mathcal{M}}}\right]+\int_{\mathcal{B}} \mathbf{C}:\left[\mathcal{L}_{\frac{\delta g}{\delta \overline{\mathcal{M}}}} \frac{\overline{\delta f}}{\delta \mathbf{C}}-\mathcal{L}_{\frac{\delta f}{\delta \overline{\mathcal{M}}}} \frac{\overline{\delta g}}{\delta \mathbf{C}}\right] \\
& -\int_{\partial_{\varphi} \mathcal{B}} \mathbf{C}:\left[\frac{\delta f}{\delta \overline{\mathcal{M}}} \otimes 2 \frac{\delta g}{\delta \mathbf{C}} \widehat{\mathbf{N}}-\frac{\delta g}{\delta \overline{\mathcal{M}}} \otimes 2 \frac{\delta f}{\delta \mathbf{C}} \widehat{\mathbf{N}}\right] \boldsymbol{\gamma}(\mathbf{C}) \\
& -\int_{\partial \mathcal{B}}\left[\left(\mathbf{C}: \frac{\delta g}{\delta \mathbf{C}}\right)\left(\frac{\delta f}{\delta \overline{\mathcal{M}}} \cdot \widehat{\mathbf{N}}\right)-\left(\mathbf{C}: \frac{\delta f}{\delta \mathbf{C}}\right)\left(\frac{\delta f}{\delta \overline{\mathcal{M}}} \cdot \widehat{\mathbf{N}}\right)\right] \boldsymbol{\gamma}(\mathbf{C}) \text {. }
\end{aligned}
$$

Proof Expression (3.27) follows from (??) by the divergence theorem. Expression (3.28) follows from (??) by the coordinate expression for the Lie derivative $\mathcal{L}_{\frac{\delta f}{\delta \overline{\mathcal{M}}}} \mathbf{C}$ and by the symmetry of $\overline{\delta f} / \delta \mathbf{C}$. Finally, expression (3.29) follows from (3.28) by integration by parts.

We observe that for pure displacement boundary conditions, the boundary term vanishes in expression (3.27) since $\partial_{\sigma} \mathcal{B}=\emptyset$, whereas for traction-free boundary conditions the boundary term in (3.28) vanishes since $\partial_{\varphi} \mathcal{B}=\emptyset$.

Corollary 3.3 Hamilton's equations $\dot{f}=\{f, H\}$ are equivalent to the convective equations of motion

$$
\begin{aligned}
\frac{\partial \overline{\mathcal{M}}}{\partial t} & =\left[\frac{1}{2} \mathcal{R} \mathbf{d}|\mathcal{V}|_{\mathbf{C}}^{2}+\left(\mathbf{D I V}_{\mathbf{C}} \boldsymbol{\Sigma}\right)^{b}\right] \otimes \mu(\mathbf{C}) \\
\frac{\partial \mathbf{C}}{\partial \mathbf{t}} & =L_{\mathcal{V}_{t}} \mathbf{C} \\
\boldsymbol{\Sigma} \widehat{\mathbf{N}} & =\mathbf{0} \quad \text { on } \partial_{\sigma} \mathcal{B} \quad\left(\text { with } \overline{\mathcal{M}}=\mathbf{0} \text { on } \partial_{\sigma} \mathcal{B}\right) .
\end{aligned}
$$

This follows, as we remarked earlier, by the general theory of reduction. For completeness we include a direct verification of this statement. 
Proof of Corollary 3.4 From (2.72), the Hamiltonian in the convective description is

$$
\mathbf{H}(\mathbf{C}, \overline{\mathcal{M}}, \mathbf{G})=\frac{1}{2} \int_{\mathcal{B}} \mathcal{R}|\mathcal{V}|_{\mathbf{C}}^{2} \mu(\mathbf{C})+\int_{\mathcal{B}} \mathcal{R} \overline{\bar{W}}(\mathbf{C}, \mathbf{G}) \mu(\mathbf{C})
$$

where $\overline{\mathcal{M}}=\mathcal{M} \otimes \mu(\mathbf{C})=\mathcal{R} \mathcal{V}^{b} \otimes \mu(\mathbf{C}) \equiv \varrho_{0} \mathcal{V}^{b} \otimes \mu(\mathbf{G})$. The partial functional derivatives are computed in the standard fashion by considering curves $\varepsilon \mapsto \mathbf{C}_{\varepsilon} \in S_{2}(\mathcal{B})$ and $\varepsilon \mapsto \overline{\mathcal{M}}_{\varepsilon} \in \mathfrak{X}^{*}(\mathcal{B})$ and using the directional derivative formula. One finds that

$$
\frac{\overline{\delta H}}{\delta \mathbf{C}}=\frac{1}{2}[-\mathcal{R} \mathcal{V} \otimes \mathcal{V}+\boldsymbol{\Sigma}] \otimes \mu(\mathbf{C}) ; \quad \frac{\delta H}{\delta \overline{\mathcal{M}}}=\mathcal{V} .
$$

Using (3.32) and the definition of the Lie bracket of vector fields in $\mathfrak{X}(\mathcal{B})$, we write the first term in $(3.28)$ as

$$
\int_{\mathcal{B}} \overline{\mathcal{M}} \cdot\left[\frac{\delta f}{\delta \overline{\mathcal{M}}}, \frac{\delta H}{\delta \overline{\mathcal{M}}}\right]=\int_{\mathcal{B}} \overline{\mathcal{M}} \cdot\left[\frac{\delta f}{\delta \overline{\mathcal{M}}}, \mathcal{V}\right]=\int_{\mathcal{B}} \overline{\mathcal{M}} \cdot \mathcal{L}_{\frac{\delta f}{\delta \overline{\mathcal{M}}}} \mathcal{V} .
$$

The second term in $(3.28)$ reduces to

$$
\int_{\mathcal{B}} \frac{\overline{\delta f}}{\delta \mathbf{C}}: \mathcal{L}_{\frac{\delta H}{\delta \overline{\mathcal{M}}}} \mathbf{C}=\int_{\mathcal{B}} \frac{\overline{\delta f}}{\overline{\delta \mathbf{C}}}: \mathcal{L}_{\mathcal{V}} \mathbf{C}
$$

The last term in (3.28) requires a more elaborate computation. First, we use the derivative property of Lie derivatives to obtain:

$$
-\int_{\mathcal{B}} \frac{\delta \bar{H}}{\delta \mathbf{C}}: \mathcal{L}_{\frac{\delta f}{\delta \overline{\mathcal{M}}}} \mathbf{C}=-\int_{\mathcal{B}} \mathcal{L}_{\frac{\delta f}{\delta \bar{M}}}\left(\mathbf{C}: \frac{\overline{\delta H}}{\delta \mathbf{C}}\right)+\int \mathbf{C}: \mathcal{L}_{\frac{\delta f}{\delta \overline{\mathcal{M}}}} \frac{\overline{\delta H}}{\delta \mathbf{C}} .
$$

The substitution of (3.32) into (3.35) and the use of standard additional properties of the Lie derivative yields

$$
\begin{aligned}
-\int_{\mathcal{B}} \frac{\overline{\delta H}}{\delta \mathbf{C}}: \mathcal{L}_{\frac{\delta f}{\delta \overline{\mathcal{M}}}} \mathbf{C}= & \int_{\mathcal{B}} \frac{1}{2} \mathcal{L}_{\frac{\delta f}{\delta \overline{\mathcal{M}}}}\left[|\mathcal{V}|_{\mathbf{C}}^{2} \otimes \mathcal{R} \mu(\mathbf{C})-(\mathbf{C}: \boldsymbol{\Sigma}) \otimes \mu(\mathbf{C})\right] \\
& -\int_{\mathcal{B}} \mathbf{C}:\left[\left(\mathcal{L}_{\frac{\delta f}{\delta \overline{\mathcal{M}}}} \mathcal{V}\right) \otimes \mathcal{V} \otimes \mathcal{R} \mu(\mathbf{C})\right]+\frac{|\mathcal{V}|_{\mathbf{C}}^{2}}{2} \otimes \mathcal{L}_{\frac{\delta f}{\delta \overline{\mathcal{M}}}}[\mathcal{R} \otimes \mu(\mathbf{C})] \\
& +\int_{\mathcal{B}} \frac{1}{2} \mathbf{C}: \mathcal{L}_{\frac{\delta f}{\delta \overline{\mathcal{M}}}}[\boldsymbol{\Sigma} \otimes \mu(\mathbf{C})] \\
= & \int_{\mathcal{B}}\left[\frac{\mathcal{R}}{2} \mathbf{d}|\mathcal{V}|_{\mathbf{C}}^{2} \mu(\mathbf{C})-\overline{\mathcal{M}} \cdot \mathcal{L}_{\frac{\delta f}{\delta \overline{\mathcal{M}}}} \mathcal{V}\right] \\
& +\frac{1}{2} \int_{\mathcal{B}}\left[\mathbf{C}: \mathcal{L}_{\frac{\delta f}{\delta \overline{\mathcal{M}}}} \boldsymbol{\Sigma}-\mathcal{L}_{\frac{\delta f}{\delta \mathcal{M}}}(\mathbf{C}: \boldsymbol{\Sigma})\right] \otimes \mu(\mathbf{C}) .
\end{aligned}
$$

By exploiting the symmetry of $\boldsymbol{\Sigma}$ and the expression in local coordinates for the Lie derivative, we obtain

$$
\begin{aligned}
\frac{1}{2} \mathbf{C}: \mathcal{L}_{\frac{\delta f}{\delta \overline{\mathcal{M}}}} \boldsymbol{\Sigma} & =\frac{1}{2} \mathbf{d}(\mathbf{C}: \boldsymbol{\Sigma}) \cdot \frac{\delta f}{\delta \overline{\mathcal{M}}}-\left(\widetilde{\nabla} \frac{\delta f}{\delta \overline{\mathcal{M}}}\right): \boldsymbol{\Sigma} \\
& =\frac{1}{2} \mathcal{L}_{\frac{\delta f}{\delta \overline{\mathcal{M}}}}(\mathbf{C}: \boldsymbol{\Sigma})-\left(\widetilde{\nabla} \frac{\delta f}{\delta \overline{\mathcal{M}}}\right): \boldsymbol{\Sigma},
\end{aligned}
$$

where in coordinates, $(\widetilde{\nabla} \delta f / \delta \overline{\mathcal{M}}): \boldsymbol{\Sigma}=\left(\delta f / \delta \overline{\mathcal{M}}_{A}\right)_{\mid B} C_{A D} \boldsymbol{\Sigma}^{D B}$. The substitution of (3.37) into (3.36), the integration of the resulting expression by parts and the use of the Gauss theorem then 
yield

$$
\begin{aligned}
-\int_{\mathcal{B}} \frac{\overline{\delta H}}{\delta \mathbf{C}}: \mathcal{L}_{\frac{\delta f}{\delta \overline{\mathcal{M}}} \mathbf{C}=} & \int_{\mathcal{B}} \frac{\delta f}{\delta \overline{\mathcal{M}}} \cdot\left[\frac{\mathcal{R}}{2} \mathbf{d}|\mathcal{V}|_{\mathbf{C}}^{2}+\mathbf{D I V}_{\left.\left.\mathbf{C}^{\boldsymbol{\Sigma}}\right)^{b}\right] \otimes \mu(\mathbf{C})}\right. \\
& +\int_{\mathcal{B}}-\mathbf{D I V}_{\mathbf{C}}\left(\boldsymbol{\Sigma} \frac{\delta f}{\delta \overline{\mathcal{M}}}\right) \otimes \mu(\mathbf{C})-\overline{\mathcal{M}} \cdot \mathcal{L}_{\frac{\delta f}{\delta \overline{\mathcal{M}}}} \mathcal{V} \\
= & \int_{\dot{\mathcal{B}}} \frac{\delta f}{\delta \overline{\mathcal{M}}} \cdot\left[\frac{\mathcal{R}}{2} \mathbf{d}|\mathcal{V}|_{\mathbf{C}}^{2}+\left(\mathbf{D I V} \mathbf{C}_{\mathbf{C}} \boldsymbol{\Sigma}\right)^{b}\right] \otimes \mu(\mathbf{C}) \\
& -\int_{\partial \mathcal{B}} \frac{\delta f}{\delta \overline{\mathcal{M}}} \cdot(\boldsymbol{\Sigma} \widehat{\mathbf{N}})^{b} \boldsymbol{\gamma}(\mathbf{C})-\int_{\mathcal{B}} \overline{\mathcal{M}} \cdot \mathcal{L}_{\frac{\delta f}{\delta \overline{\mathcal{M}}}} \mathcal{V} .
\end{aligned}
$$

Substituting (3.33), (3.34) and (3.38) into (3.28) yields

$$
\begin{aligned}
\{f, H\}= & \int_{\mathcal{B}} \frac{\overline{\delta f}}{\delta \overline{\mathbf{C}}}: \mathcal{L}_{\mathcal{V}} \mathbf{C}+\frac{\delta f}{\delta \overline{\mathcal{M}}} \cdot\left[\frac{\mathcal{R}}{2} \mathbf{d}|\mathcal{V}|_{\mathbf{C}}^{2}+\left(\mathbf{D I V}_{\mathbf{C}} \boldsymbol{\Sigma}\right)^{b}\right] \otimes \mu(\mathbf{C}) \\
& -\int_{\partial \mathcal{B}} \frac{\delta f}{\delta \overline{\mathcal{M}}} \cdot(\boldsymbol{\Sigma} \widehat{\mathbf{N}})^{b} \boldsymbol{\gamma}(\mathbf{C}) \\
& -\int_{\partial_{\varphi} \mathcal{B}}\left[\mathcal{V} \cdot\left(2 \frac{\delta f}{\delta \mathbf{C}} \widehat{\mathbf{N}}\right)^{b}-\frac{\delta f}{\delta \overline{\mathcal{M}}} \cdot(\boldsymbol{\Sigma} \widehat{\mathbf{N}})^{b}-\mathcal{M}(\mathcal{V} \cdot \widehat{\mathbf{N}})\right] \gamma(\mathbf{C}) .
\end{aligned}
$$

Since $\mathcal{V} \mid \partial_{\varphi} \mathcal{B}=\mathbf{0}$, the boundary term in (3.39) collapes to

$$
-\int_{\partial_{\sigma} \mathcal{B}} \frac{\delta f}{\delta \overline{\mathcal{M}}} \cdot(\boldsymbol{\Sigma} \widehat{\mathbf{N}})^{b} \gamma(\mathbf{C}) .
$$

On the other hand, for any $f: \mathcal{P}_{\text {conv }} \rightarrow \mathbb{R}$, we have

$$
\dot{f}=\int_{\mathcal{B}} \frac{\overline{\delta f}}{\delta \mathbf{C}}: \dot{\mathbf{C}}+\frac{\delta f}{\delta \mathcal{M}} \cdot \dot{\bar{M}} .
$$

A comparison of (3.39)-(3.40) with (3.41) yields the desired result. 